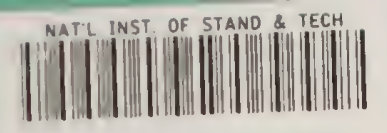

A $11706 \quad 053726$

NBSIR $85-3234$

Dynamic Green's Functions of an Infinite Plate - A Computer Program

$\begin{array}{cc}\text { Reference } & \text { NBS } \\ \text { PUBLICATIONS }\end{array}$

iNelson N. Hisu

U.S. DEPARTMENT OF COMMERCE

ivational Bureau of Standards

National Engireering Laboratory

Center for Manufacturing Engineering

Gaithersburg, MD 20899

August 1985

issued November 1985

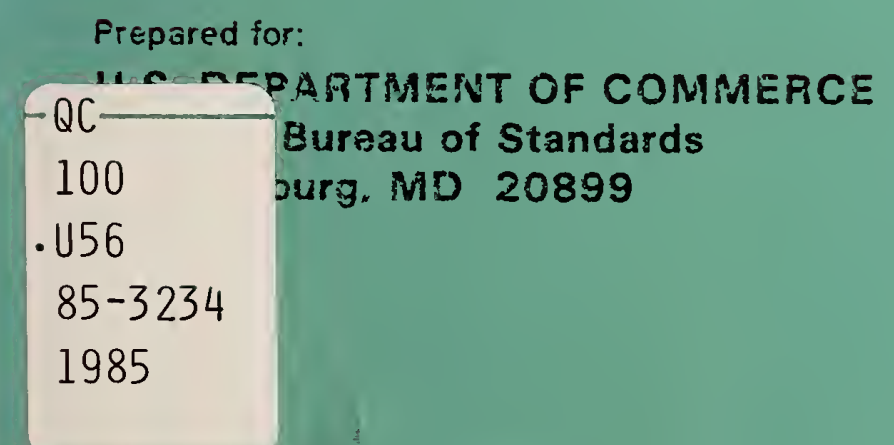





\section{DYNAMIC GREEN'S FUNCTIONS OF AN INFINITE PLATE - A COMPUTER \\ PROGRAM}

Nelson N. Hsu

U.S. DEPARTMENT OF COMMERCE

National Bureau of Standards

National Engineering Laboratory

Center for Manufacturing Engineering

Gaithersburg, MD 20899

\section{August 1985}

Issued November 1985

Prepared for:

U.S. DEPARTMENT OF COMMERCE

National Bureau of Standards

Gaithersburg, MD 20899

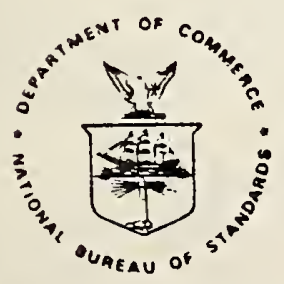

U.S. DEPARTMENT OF COMMERCE, Malcolm Baldrige, Secretary

NATIONAL BUREAU OF STANDARDS, Ernest Ambler, Director 

Dynamic Green's Functions of an Infinite Plate - a Computer Program

Nelson N. Hsu

National Bureau of Standards

Gaithersburg, MD 20899 USA

\section{Introduction}

This report is a FORTRAN program to compute the Green's functions of an infinite plate. The Green's function, $G_{i j}(\underline{\xi}, \underline{x}, t)$, is defined as the ith component of the displacement at $x$ due to a joint force of step-function time dependency acting at $\underline{\xi}$ in the $j$ th direction initiated at $t=0$. The Green's function is the fundamental solution of the transient elastic wave propagation problem. In general, the displacement field $\underline{u}(\underline{\xi}, \underline{x}, t)$ at $\underline{x}$ due to a point force of arbitrary time dependence acting at $\underline{\xi}$ can $\bar{b} e$ computed $\bar{b} y$ a convolution integration; i.e.,

$$
u_{i}(\underline{\xi}, \underline{x}, t)=\int_{0}^{\infty} G_{i j}(\underline{\xi}, \underline{x}, t) f_{j}(\tau-t) d \tau .
$$

Here, $G_{i j}$ is the time derivative of $G_{i j}$ and $f_{j}(t)$ is the point force component of arbitrary time dependence acting $i \frac{1}{j}$ the $j$ th direction (summation over repeated indices is used). Displacement due to point dipoles or couple forces can be represented by the spatial derivatives of $G_{i j}$. Displacement produced by a dynamic force distributed over a finite area can also be computed by numerical integration using the Green's function as the kernel of the integral over the finite area.

The basic formulation of the problem and derivation of the solution formulas were reported in (1). The method used is called "ray theory" in the seismological literature. Our derivation was based upon John Willis' new Fourier Inversion method (2). Similar computation results can be found in Reference (3), (4), and (5).

The program was originally written for the analysis of acoustic emission signals. Acoustic emission is a nondestructive testing and monitoring technique in which the detection of the transient stress wave produced by localized deformation or cracks are used to locate, and to assess the criticality of the defects. The theoretical computation is an important link to predict how the acoustic emission waveform evolves through the structure. How the theoretical computations are used in the study of acoustic emission can be found in References (1), (6), (7), and (8).

This computer program is made available mainly for its application to calibrate acoustic emission systems and sensors. By making the present program available, duplication of efforts can be avoided, errors in theory can be checked, and experimental results can be reproduced and verified.

How to Use the Program

I. The easiest way to run this program is by setting up nondimensional parameters and calling the subroutine GREENFCT. First, the $x-y-z$ coordinates 
are selected by choosing an $x-y$ plane parallel to the plate and the origin at the center of the plate directly underneath the source, i.e., the source is always located at $\xi=(0,0,0.5)$; the $x$ axis is aligned in the direction from the source pointing to the detector. The required nondimensionalized input parameters for the subroutine GREENFCT are:

1. ALPHA = shear wave speed/longitudinal wave speed;

2. $X D=x$-coordinate of the detector; actual length/thickness of the plate;

3. $Z D=z$-coordinate of the detector; actual length/thickness of the plate. $Z D=0.5$ if the source and the detector are on the same side of the plate;

$Z D=-0.5$ if they are on opposite sides of the plate.

(Note: the $y$-coordinate of the detector is always zero because of the way the coordinate system is chosen.)

4. INDEX = ij; subscript of Green's function, integer number $11,12,13,21$, $22,23,31,32$, or 33 . (111, 112, 113, etc. for force dipoles.)

5. TDELTA = sampling time interval in terms of nondimensionalized time unit = actual sampling time interval * shear wave speed/thickness of the plate; and

6. NPOINT = Total number of sampling points to be computed; must be an integer.

The subroutine will return a double precision array DISPL of dimension (NPOINT) which corresponds to the desired $G_{i j}(\underline{\xi}, \underline{x}, t)$ or $G_{i j}(\underline{\xi}, \underline{x}, t)$ sampled at equal time intervals. However, the displaceithents corresponding to dipoles or couples (i.e., $G_{i j k}$ ) are for linear ramp time dependency input. Differentitating the returned DISPL with respect to time once will give the proper Green's function due to step time dependency input.

A simple program calls GREENFCT for given nondimensionalized input paramters together with the output results is included in Appendix A; a program that prompts the user for input parameters of arbitrary physical units and returns displacement in physical units is included in Appendix B. The complete listing of the subroutines is in Appexdix $C$.

II. Another way to use this program is by calling GREENSUB which is basically a simple function: for given one time $T$, it returns a displacement DISPL.

However, there are two preparatory steps that must be taken in the main program:

(1) RAYTIME (NRAY, 3), the time of arrival table, TA(NRAY) and CN(NRAY, 3), two working arrays, should be dimensioned.

(2) The subroutines INIT and TIMEARRI must be called first.

The subroutine GREENFCT may serve as an example of how to use the subroutine GREENSUB.

III. In addition to the two subroutines mentioned above, there are three subroutines which may be called independently and the user may $f$ ind them useful in checking experimental or theoretical results. 
(1) EPIDIS computes the vertical displacement at the epicenter for given ALPHA and $T$.

(2) SFWAVE computes the surface wave, which corresponds to the Green's function of a semi-infinite space.

(3) TIMEARRI computes the time of arrival for various ray paths.

Comments in these three subroutines serve as instructions about how to use these subroutines.

Some Remarks

I. There are two machine dependent constants which are used in the two numerical integration routines DGLQI and GLI5T. EPMACH is the largest relative spacing. UFLOW is the smallest positive magnitude. See Reference (9) for details about how to set these constants for different computers.

II. The program uses double precision complex arithmetic. In order to run on those FORTRAN compilers lacking double precision complex function libraries, the user may have to modify the program to single precision arithmetic.

III. The ray method is an exact solution for the Green's function in the time domain produced by summing the contribution from successive arrivals of reflected rays. The numerical computation is accurate if the source and detector are near each other (say less than ten plate thicknesses) and if the maximum time is less than ten dimensionless units. If the sensor and detector are far apart or the maximum time is large, the number of rays arriving at approximately the same time may be too large to compute in a reasonable time and the accumulated error may grow.

IV. The current program is limited to the test configuration where the sensor and detector are on the surface of the plate. The displacement computed for force dipole (i.e., INDEX $=111,112$, etc.) are for point dipole of linear ramp time dependency. Differentiation with respect to time once of the displacement will produce proper Green's functions due to step time dependency (See Reference $10)$.

Acknowledgement

The author would like to thank Dr. David Kahaner of the Scientific Computing Division, NBS, for supplying the integration subroutines DGLQ and GLIST .

References:

1. D.G. Eitzen, et. al., "Fundamental Developments for Quantitative Acoustic Emission Measurements," EPRI NP-2089, Project 608-1, Palo Alto, CA:

Electric Power Research Institute, Oct. 1981.

2. J.R. Willis, "Self-Similar Problems in Elastodynamics," Phil. Trans. of Roy. Soc. London, 274, pp. 435-491, May 1973. 
3. Y. Pao, Ralph R. Gajewski and Anmet N. Ceranouglu, "Acoustic Emission and Transient Waves in an Elastic Plate," J. Acoust. Soc. Am., 65(1), Jan. 1979 .

4. A.N. Cernoglu and Y.H. Pao, "Propagation of Eleastic Pulses ard Acoustic Emission in a Plate," ASME Journal of Applied Mechanics, 48, pp. 125-147. Mar. 1981.

5. T.M. Proctor, F.R. Breckenridge and Y.H. Pao, "Transient Waves in an Elastic Plate: Theory and Experiment Compared," J. Acoust. Soc. Am., (6) Dec. 1983.

6. N.N. Hsu, J.A. Simmons and S.C. Hardy, "An Approach to Acoustic Emission Signal Analysis -- Theory and Experiment," Materials Evaluation, 35, pp. 100-106, Oct. 1977 .

7. N.N. Hsu and S.C. Hardy, "Experiments in Acoustic Emission Waveform Analysis for Characterization of AE Sources, Sensors and Structures; In Elastic Waves and Non-Destructive Testing of Materials," AMD-Vol. 29, Y.H. Pao, Editor, The American Society of Mechanical Engineers, New York, pp. 85-106, Dec. 1978.

8. C. Chang and W. Sachse, "Analysis of Elastic Wave Signals from An Extended Source in A Plate," J. Acoust. Soc. Am., 77(4), April 1985.

9. P.A. Fox, A.D. Hall and N.L. Schryer, Bell Laboratories Computer Science Technical Report No. 47. Or: The PORT Mathematical Subroutine Library, ACM Transactions on Mathematical Software, Vol. 4, No. 2, pp 104-106.

10. J.E. Sinclair, "Epicentre Solutions for Point Multipole Sources in An Elastic Half-Space," J. Phys. D: Appl. Phys., Vol. 12, 1979, pp 1309-1315. 
Appendix A

Sample Program and Results 


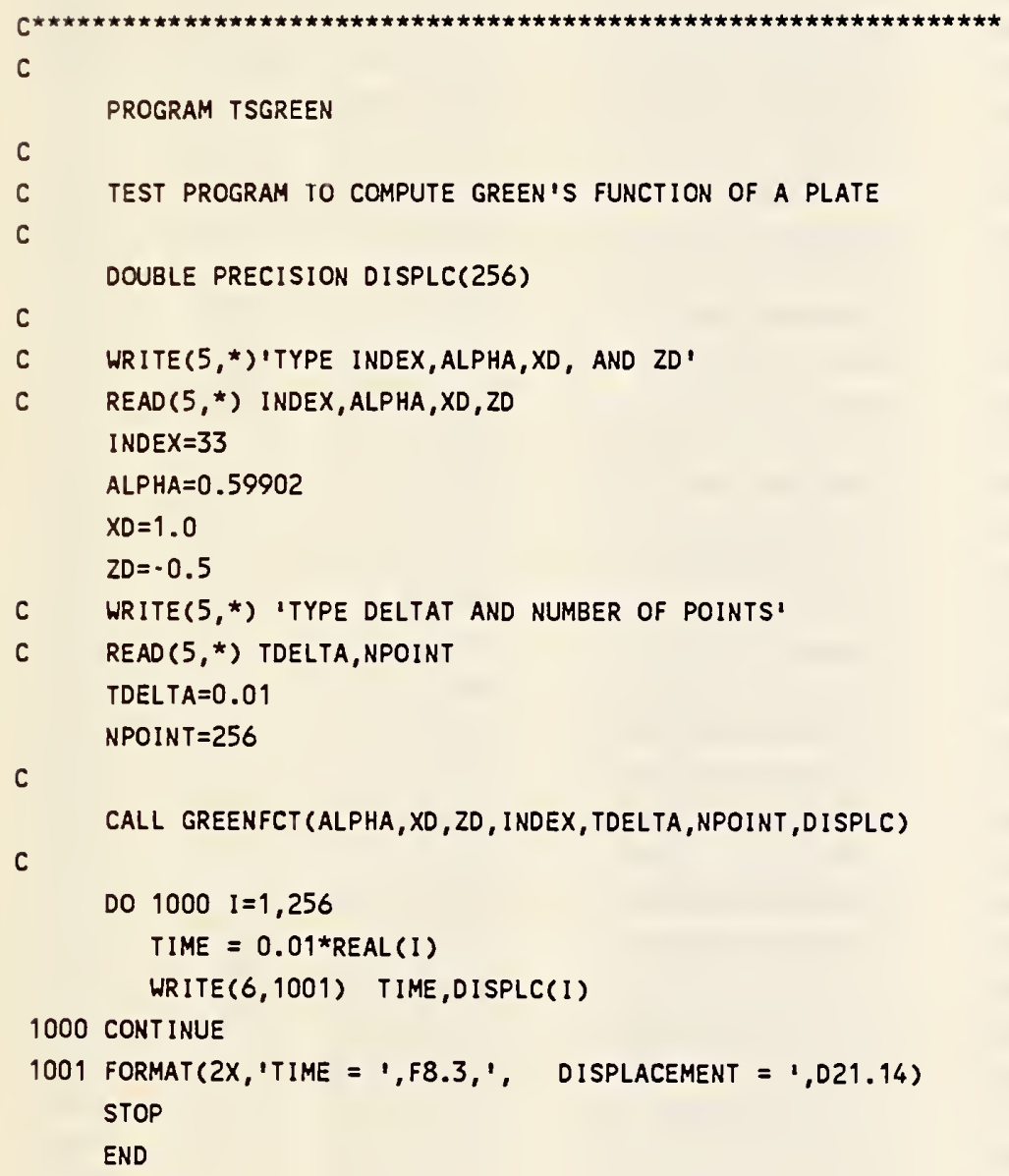




\begin{tabular}{|c|c|c|c|}
\hline & & CEMENT $=$ & \\
\hline & & ISPLACEMENT $=$ & $0.00000000000000 \mathrm{D}+00$ \\
\hline & .030, & ISPLACEMENT = & $0.00000000000000 \mathrm{D}+00$ \\
\hline IE & .040 & I SPLACEMENT $=$ & $0.00000000000000 D+00$ \\
\hline & .050, & SPLACEMENT $=$ & $0.000000000000000+00$ \\
\hline$E=$ & .060 & ISPLACEMENT $=$ & $0.00000000000000 D+00$ \\
\hline ME $=$ & -070, & SPLACEMENT $=$ & $0.00000000000000 \mathrm{D}+00$ \\
\hline & & ISPLACEMENT $=$ & $0.00000000000000 \mathrm{D}+00$ \\
\hline ME & & I SPLACEMENT & $0.00000000000000 \mathrm{D}+00$ \\
\hline ME & & I SPLACEMENT & $0.000000000000000+00$ \\
\hline ME & & I SPLACEMENT $=$ & $0.00000000000000 \mathrm{D}+00$ \\
\hline$M E=$ & .120 & ISPLACEMENT = & $0.00000000000000 \mathrm{D}+00$ \\
\hline ME $=$ & 30, & I SPLACEMENT = & $0 D+00$ \\
\hline ME & & CEMENT & $D+00$ \\
\hline ME & & I SPLACEMENT & $0.00000000000000 \mathrm{D}+00$ \\
\hline IME & & ISPLACEMENT $=$ & $0.000000000000000+00$ \\
\hline ME & & I SPLACEMENT $=$ & $0.00000000000000 D+00$ \\
\hline ME & 180, & ISPLACEMENT = & 0.000 \\
\hline ME & .190, & I SPLACEMENT $=$ & 0.000 \\
\hline IME & , & EMENT & $D+\infty 0$ \\
\hline ME & & I SPLACEMEN & $0.00000000000000 D+00$ \\
\hline IME & & DI SPLACEMENT & $0.00000000000000 \mathrm{D}+00$ \\
\hline ME & & SPLACEMENT & $0.00000000000000 \mathrm{D}+00$ \\
\hline ME & 40 , & SPLACEMENT & $+\infty 0$ \\
\hline IME $=$ & .250, & ISPLACEMENT $=$ & $00+00$ \\
\hline IME & & ISPLACEMENT & $0.00000000000000 D+00$ \\
\hline ME & & I SPLACEMENT & $0.000000000000000+00$ \\
\hline ME & 0.2 & ISPLACEMENT = & 0.0000000000 \\
\hline & & ISPLACEMENT & 0.0000 \\
\hline$M E=$ & & SPLACEMENT $=$ & 0.000 \\
\hline IME $=$ & & ISPLACEMEN & +00 \\
\hline ME & & I SPLACEMENT & $0.00000000000000 D+00$ \\
\hline & & SPLACEMENT & $0.00000000000000 \mathrm{D}+00$ \\
\hline & & CEMENT & \\
\hline ME & & SPLACEMENT $=$ & $00000+00$ \\
\hline IME $=$ & & ISPLACEMENT $=$ & $000+00$ \\
\hline$M E=$ & & & $0.000000000000000+00$ \\
\hline$M E$ & & SPLACEMENT & 0.00 \\
\hline & & CEMENT $=$ & 0.0000 \\
\hline ME $=$ & & SPLACEMENT $=$ & 0.000 \\
\hline $\mathrm{ME}=$ & & SPLACEMENT $=$ & 0.00 \\
\hline$M E=$ & & SPLACEMENT $=$ & $0.00000000000000 D+00$ \\
\hline ME : & & CEMENT $=$ & $0.000000000000000+00$ \\
\hline ME & & I SPLACEMENT & $0.000000000000000+00$ \\
\hline & & SPLACEMENT & $0.00000000000000 D+00$ \\
\hline AE & & ISPLACEMENT = & $0.0000000000000000+00$ \\
\hline ME $=$ & 0 , & ISPLACEMENT = & $0.000000000000000+00$ \\
\hline ME $=$ & & ISPLACEMENT = & $0.000000000000000+00$ \\
\hline & & DISPLACEMENT $=$ & $0.000000000000000+00$ \\
\hline & & DISPLACEMENT $=$ & $0.000000000000000+00$ \\
\hline & & DISPLACEMEN & $0.00000000000000 D+00$ \\
\hline & & DI SPLACEMENT & $0.000000000000000+00$ \\
\hline & & DISPLACEMENT & $0.000000000000000+00$ \\
\hline & & DI & $0.00000000000000 \mathrm{D}+00$ \\
\hline
\end{tabular}




\begin{tabular}{|c|c|c|c|c|c|}
\hline IME & $=$ & 0.550, & DI SPLACEMENT & $=$ & $0.000000000000000+00$ \\
\hline ME & $=$ & .560 & ISPLACEMENT & $=$ & $0.000000000000000+00$ \\
\hline ME & $=$ & .570 & I SPLACEMENT & $=$ & $0.000000000000000+00$ \\
\hline ME & $=$ & .580 & ISPLACEMENT & $=$ & $0.000000000000000+00$ \\
\hline ME & $=$ & .590 & I SPLACEMENT & $=$ & $0.000000000000000+00$ \\
\hline ME & $=$ & .600 & I SPLACEMENT & $=$ & $0.00000000000000 D+00$ \\
\hline IME & $=$ & .610 & ISPLACEMENT & $=$ & $0.000000000000000+00$ \\
\hline IME & $=$ & & ISPLACEMENT & $=$ & $0.000000000000000+00$ \\
\hline TIME & $=$ & .630 & I SPLACEMENT & $=$ & $0.000000000000000+00$ \\
\hline TIME & $=$ & .640 & I SPLACEMENT & $=$ & $0.000000000000000+00$ \\
\hline MF & $=$ & .650 & I SPLACEMENT & $=$ & $0.000000000000000+00$ \\
\hline TIME & $=$ & 60, & ISPLACEMENT & $=$ & $0.000000000000000+00$ \\
\hline TIME & $=$ & .670 & ISPLACEMENT & $=$ & $0.000000000000000+00$ \\
\hline TIME & $=$ & .680, & ISPLACEMENT & $=$ & $0.000000000000000+00$ \\
\hline TIME & $=$ & .690 , & I SPLACEMENT & $=$ & $0.000000000000000+00$ \\
\hline TIME & $=$ & .700 & I SPLACEMENT & $=$ & $0.000000000000000+00$ \\
\hline TIME & $=$ & .710 & I SPLACEMENT & $=$ & $0.000000000000000+00$ \\
\hline TIME & $=$ & & I SPLACEMENT & $=$ & $0.000000000000000+00$ \\
\hline TIME & $=$ & 30, & I SPLACEMENT & $=$ & $0.000000000000000+00$ \\
\hline TIME & $=$ & 40, & I SPLACEMENT & $=$ & $0.000000000000000+00$ \\
\hline TIME & $=$ & & I SPLACEMENT & $=$ & $000000 D+00$ \\
\hline TIME & $=$ & & I SPLACEMENT & $=$ & $0 D+00$ \\
\hline TIME & $=$ & & I SPLACEMENT & $=$ & $0.000000000000000+00$ \\
\hline TIME & $=$ & & I SPLACEMENT & $=$ & $00+00$ \\
\hline TIME & $=$ & & I SPLACEMENT & $=$ & $0 D+00$ \\
\hline TIME & $=$ & .800 & DI SPLACEMENT & $=$ & $D+00$ \\
\hline TIME & $=$ & .810 & I SPLACEMENT & $=$ & $0 D+00$ \\
\hline TIME & $=$ & 0.820 & DISPLACEMENT & $=$ & $0 D+00$ \\
\hline TIME & $=$ & 0.8 & ISPLACEMENT & $=$ & $0 D+00$ \\
\hline TIME & $=$ & 0.840 & I SPLACEMENT & $=$ & $D+00$ \\
\hline TIME & $=$ & 0.850 & ISPLACEMENT & $=$ & $20+00$ \\
\hline TIME & $=$ & 0.860 & DI SPLACEMENT & $=$ & $2 D+00$ \\
\hline TIME & $=$ & 0.870 & DISPLACEMENT & $=$ & $70+00$ \\
\hline TIME & $=$ & 0.880 & DISPLACEMENT & $=$ & $482 D+00$ \\
\hline TIME & $=$ & 0.890, & DISPLACEMENT & $=$ & $0.11144318482884 D+00$ \\
\hline TIME & $=$ & 0.900 & DISPLACEMENT & $=$ & $1102652121 D+00$ \\
\hline TIME & $=$ & 0.910 & DISPLACEMENT & $=$ & $0.11153587694415 D+00$ \\
\hline TIME & $=$ & .920 & DISPLACEMENT & $=$ & $0.111517336058210+00$ \\
\hline TIME & $=$ & 0.930 & DICOI ACEMEUT & $=$ & $0.111455035416960+00$ \\
\hline TIME & $=$ & 0.940 & & $=$ & \\
\hline TIME & $=$ & 0.950 & & $=$ & $0.111197870207070+00$ \\
\hline TIME & $=$ & & & $=$ & $0.111002474721780+00$ \\
\hline TIME & $=$ & & DISPLACEMENT & $=$ & $0.11076226552545 D+00$ \\
\hline TIME & $=$ & 0.9 & DISPLACEMENT & $=$ & $0.110477107642190+00$ \\
\hline TIME & $=$ & 0.9 & DISPLACEMENT & $=$ & $0.110146923949600+00$ \\
\hline TIME & $=$ & 1.000 & DISPLACEMENT & $=$ & $0.109771714297700+00$ \\
\hline TIME & $=$ & 1.010, & DISPLACEMENT & $=$ & $0.109351509141280+00$ \\
\hline TIME & $=$ & 1.0 & DISPLACEMENT & $=$ & $0.10888645033515 D+00$ \\
\hline TIME & $=$ & 1.030, & DI SPLACEMENT & $=$ & $0.108376789796460+00$ \\
\hline TIME & $=$ & 1.040, & DISPLACEMENT & $=$ & $0.107822708452880+00$ \\
\hline TIME & $=$ & 1.050, & DISPLACEMENT & $=$ & $0.10722461062043 D+00$ \\
\hline TIME & $=$ & 1.060, & DISPLACEMENT & $=$ & $0.106582936232970+00$ \\
\hline TIME & $=$ & 1.070, & DISPLACEMENT & $=$ & $0.10589820949205 \mathrm{D}+00$ \\
\hline TIME & $=$ & 1.080, & DISPLACEMENT & $=$ & $0.105171112108310+00$ \\
\hline TIME & $=$ & 1.090, & DISPLACEMENT & $=$ & $0.104402203460990+00$ \\
\hline
\end{tabular}




\begin{tabular}{|c|c|c|c|}
\hline & & ISPLACEMENT $=$ & \\
\hline ME & 1.110, & I SPLACEMENT $=$ & $0.102742393320290+00$ \\
\hline & & ISPLACEMENT $=$ & $0.101853423885000+00$ \\
\hline & & ISPLACEMENT $=$ & $0.100926319463770+00$ \\
\hline ME & .140, & DISPLACEMENT $=$ & $0.99962313600975 \mathrm{D}-01$ \\
\hline MEE $=$ & & DISPLACEMENT $=$ & $0.989626479399810-01$ \\
\hline IME $=$ & & ISPLACEMENT = & $0.979287547859510-01$ \\
\hline ME & & ICEMENT $=$ & $90-01$ \\
\hline IME $=$ & & DISPLACEMENT $=$ & $164670-01$ \\
\hline IME $=$ & & DISPLACEMENT $=$ & $0.946354742077300 \cdot 01$ \\
\hline IME $=$ & 1.200 & DISPLACEMENT $=$ & $0.934793763548660-01$ \\
\hline IME $=$ & & ISPLACEMENT $=$ & $0-01$ \\
\hline IME $=$ & .220 & ISPLACEMENT $=$ & \\
\hline & & CEMENT $=$ & .01 \\
\hline$M E=$ & & DISPLACEMENT $=$ & $25550-01$ \\
\hline IME & & DISPLACEMENT $=$ & $17540-01$ \\
\hline IME $=$ & 1.260, & DISPLACEMENT $=$ & $50-01$ \\
\hline MEE $=$ & & DISPLACEMENT $=$ & .01 \\
\hline$M E=$ & .280, & ISPLACEMENT $=$ & \\
\hline ME $=$ & 290, & ISPLACEMENT $=$ & \\
\hline IME $=$ & & DISPLACEMENT $=$ & -01 \\
\hline IME & & DISPLACEMENT $=$ & -01 \\
\hline I ME & & DISPLACEMENT $=$ & $90-01$ \\
\hline IME & & PLACEMENT $=$ & \\
\hline IME $=$ & & ACEMENT $=$ & \\
\hline IME $=$ & 50 , & DISPLACEMENT $=$ & \\
\hline IME $=$ & 60, & DLACEMENT $=$ & -01 \\
\hline IME $=$ & & DISPLACEMENT $=$ & -01 \\
\hline IME & & DISPLACEMENT $=$ & $1-01$ \\
\hline IME $=$ & , & LACEMENT $=$ & \\
\hline$E=$ & ， & ISPLACEMENT = & \\
\hline IME $=$ & .410, & DISPLACEMENT $=$ & -0.3 \\
\hline IME $=$ & 420, & DISPLACEMENT $=$ & \\
\hline IME $=$ & & ACEMENT $=$ & \\
\hline IME & 0 , & ACEMENT $=$ & \\
\hline IME $=$ & .450, & MENT $=$ & \\
\hline IME $=$ & 460, & ISPLACEMENT $=$ & $+\infty$ \\
\hline IME $=$ & o, & DISPLACEMENT $=$ & $0+\infty$ \\
\hline$M E=$ & & DISPLACEMEN & $0+\infty$ \\
\hline IME & & DISPLACEMENT & +00 \\
\hline IME $=$ & & ISPLACEMENT $=$ & 0.49 \\
\hline IME $=$ & & DISPLACEMENT $=$ & \\
\hline ME $=$ & ， & ISPLACEMENT $=$ & $D+00$ \\
\hline IME $=$ & & DISPLACEMENT $=$ & $50+00$ \\
\hline I ME & & I SPLACEMEN & $190+00$ \\
\hline IME $=$ & & ISPLACEMENT $=$ & $2671341780+00$ \\
\hline IME & 1.560, & ISPLACEMENT $=$ & $D+00$ \\
\hline ME & & ISPLACEMENT & $070+00$ \\
\hline ME & & ISPLACEMENT & $9930+00$ \\
\hline & & I SPLACEMENT & $0.597095366361700+00$ \\
\hline & & DISPLACEMENT $=$ & $0.606989899510390+00$ \\
\hline ME & & DISPLACEMENT $=$ & $0.61661997278485 \mathrm{D}+00$ \\
\hline & & DI SPLACEMENT & 0.62598797 \\
\hline & & & \\
\hline & & ISPI ACEMENT & $0.64395545056472 \mathrm{D}+00$ \\
\hline
\end{tabular}




\begin{tabular}{|c|c|c|c|}
\hline & & ISPLACEMENT $=$ & \\
\hline & & SPLACEMENT $=$ & $0.66091813088975 \mathrm{D}+00$ \\
\hline & & I SPLACEMENT $=$ & $0.669032557171670+00$ \\
\hline$M E=$ & & DISPLACEMENT = & $0.676907286263710+00$ \\
\hline$M E=$ & & ISPLACEMENT = & $0.68454639170679 \mathrm{D}+00$ \\
\hline $\mathrm{AE}=$ & & I SPLACEMENT = & $0.69195338607382 \mathrm{D}+00$ \\
\hline & & I SPLACEMENT $=$ & $0.69913401239547 \mathrm{D}+00$ \\
\hline$M E=$ & & DI SPLACEMENT $=$ & $0.706091992259960+00$ \\
\hline$M E=$ & & I SPLACEMENT $=$ & $0.712831858301270+00$ \\
\hline$M E=$ & & CEMENT $=$ & $0.719358221547850+00$ \\
\hline & & ISPLACEMENT $=$ & $0.72567516353530 D+00$ \\
\hline $\mathrm{ME}=$ & & EMENT $=$ & $1890+00$ \\
\hline ME $=$ & & I SPLACEMENT = & $0.737702708981970+00$ \\
\hline ME $=$ & & MENT $=$ & $0.743422233260310+00$ \\
\hline & 1.790, & ISPLACEMENT = & $0.748951449936790+00$ \\
\hline$M E=$ & & ISPLACEMENT = & $69310+00$ \\
\hline$M E=$ & & EMENT $=$ & $460690539690+00$ \\
\hline$A E=$ & & MENT $=$ & $43430+00$ \\
\hline & , & ISPLACEMENT $=$ & $+\infty$ \\
\hline $1 E=$ & & MENT $=$ & $1+00$ \\
\hline$M E=$ & & MENT $=$ & $D+00$ \\
\hline$M E=$ & & MENT $=$ & $+\infty$ \\
\hline & 0 , & MENT = & $+\infty 0$ \\
\hline$M E=$ & & MENT $=$ & $D+00$ \\
\hline ME & & MENT = & $D+00$ \\
\hline ME & & MENT $=$ & +00 \\
\hline $\mathcal{M}=$ & & MENT $=$ & \\
\hline ME $=$ & & EMENT $=$ & +00 \\
\hline$M E=$ & & MENT $=$ & $+\infty 0$ \\
\hline ME & & MENT $=$ & $D+00$ \\
\hline $1 E=$ & & MENT $=$ & +00 \\
\hline ME $=$ & & MENT = & \\
\hline ME $=$ & & MENT = & +00 \\
\hline & & MENT : & $D+00$ \\
\hline$A E=$ & & AENT $=$ & \\
\hline ME $=$ & & MENT & \\
\hline $\mathrm{ME}=$ & & EMENT $=$ & +00 \\
\hline & & EMENT & $0+00$ \\
\hline$A E=$ & & MENT $=$ & \\
\hline$M E=$ & & MENT $=$ & \\
\hline$M E=$ & & EMENT $=$ & $0+00$ \\
\hline & & DISPI & $D+00$ \\
\hline NE = & & DISPLA & $80+00$ \\
\hline MEE $=$ & 2.08 & DISPLAC & 0.959 \\
\hline ME $=$ & & DISPLACEMENT $=$ & $D+00$ \\
\hline & & DI SPLACEMENT & $60+00$ \\
\hline & & DISPLACEMENT & $0.973209131822660+00$ \\
\hline ME $=$ & 2.12 & DISPLA & $877580920+00$ \\
\hline & & DISPLACEMENT & $650+00$ \\
\hline & & DISPLACEMENT & $187638821 D+00$ \\
\hline & & DISPLACEMENT $=$ & $0.99159818288714 D+00$ \\
\hline & & DISPLACEMENT $=$ & 0.99 \\
\hline & & ICDI ACEMEM & $07641987912 D+01$ \\
\hline & & DI SPLACEM & \\
\hline & & DI SPLACEMENT & $0.10099393168333 D+01$ \\
\hline
\end{tabular}




\begin{tabular}{|c|c|c|c|}
\hline & & SPLACEMENT $=$ & \\
\hline & .210, & ISPLACEMENT $=$ & $0.101914205758580+01$ \\
\hline & .220 & ISPLACEMENT $=$ & $0.102375997740670+01$ \\
\hline & & ISPLACEMENT $=$ & $0.102839177129060+01$ \\
\hline & 2.240 & I SPLACEMENT & $0.10330396140693 D+01$ \\
\hline ME $=$ & .250 & ISPLACEMENT & $0.103770518453790+01$ \\
\hline ME $=$ & .260, & ISPLACEMENT $=$ & $0.104239144893340+01$ \\
\hline & .270, & ISPLACEMENT = & $0.10470999932314 D+01$ \\
\hline & & I SPLACEMENT & $0.105183280291710+01$ \\
\hline IME & & I ISPLACEMENT & $0.105659136556950+01$ \\
\hline IME & 2.300, & I SPLACEMENT & $0.106137849050690+01$ \\
\hline IME & .310, & ISPLACEMENT $=$ & $0.11476631005725 D+01$ \\
\hline ME & .320 & SPLACEMENT $=$ & $56957670+01$ \\
\hline ME & .330, & CEMENT $=$ & $D+01$ \\
\hline ME & & I SPLACEMENT & $301910 D+01$ \\
\hline IME & & I SPLACEMENT & $0.117076216046980+01$ \\
\hline IME & 2.360, & ISPLACEMENT $=$ & $47580+01$ \\
\hline ME & & SPLACEMENT $=$ & +01 \\
\hline ME & .380 & ISPLACEMENT = & +01 \\
\hline ME & 390, & ACEMENT $=$ & \\
\hline ME & & ISPLACEMENT $=$ & $92900+01$ \\
\hline IME & 2.4 & ISPLACEMENT $=$ & $5800+01$ \\
\hline ME & 2.420 & ISPLACEMENT $=$ & \\
\hline ME & .430, & ISPLACEMENT = & +01 \\
\hline ME $=$ & 40 & SPLACEMENT $=$ & \\
\hline$M E=$ & & ISPLACEMENT = & $D+01$ \\
\hline ME & 2.460, & ISPLACEMENT = & 0.12 \\
\hline ME & 2.470, & ISPLACEMENT $=$ & 0.12 \\
\hline ME & 80, & SPLACEMENT & \\
\hline IME $=$ & 2.490, & I SPLACEMENT $=$ & $352 D+01$ \\
\hline$M E=$ & 2.500, & I SPLACEMENT $=$ & \\
\hline $\mathrm{ME}=$ & & CEMENT $=$ & \\
\hline ME & 2.5 & ICEMENT $=$ & $9860+01$ \\
\hline ME & 2.530 & LACEMENT $=$ & +01 \\
\hline ME & & ACEMENT $=$ & 0.13 \\
\hline ME $=$ & .550 & SPLACEMENT $=$ & $7270+01$ \\
\hline ME $=$ & 2.560 & ISPLACEMENT $=$ & $46820+01$ \\
\hline $\mathrm{ME}=$ & 2.08 & ISPLACEMENT $=$ & $2610+00$ \\
\hline & & CEMENT $=$ & $0+00$ \\
\hline$M E=$ & 2.100 & ISPLACEMENT $=$ & $960+00$ \\
\hline ME & 2.110, & ISPLACEMENT = & 0.973 \\
\hline ME $=$ & .120 & ISPLACEMENT $=$ & 0.977 \\
\hline ME $=$ & 2.130, & DISPLACEMENT $=$ & $3450+00$ \\
\hline & & DISPLACEMENT $=$ & $8210+00$ \\
\hline & & DISPLACEMENT $=$ & $8714 D+00$ \\
\hline IME & 2.160, & ISPLACEMENT $=$ & $8300+00$ \\
\hline ME & 2.170, & DISPLACEMENT $=$ & 0.100 \\
\hline & 2.180, & ISPLACEMENT = & $5950+01$ \\
\hline TIME $=$ & & DISPLACEMENT $=$ & $68333 D+01$ \\
\hline & & DISPLACEMENT $=$ & $0.101453622377500+01$ \\
\hline & & DISPLACEMENT $=$ & $0.10191420575858 D+01$ \\
\hline & 2.220, & DISPLACEMENT $=$ & $740670+01$ \\
\hline & & ISPLACEMENT & $0.102839177129060+01$ \\
\hline & & & \\
\hline & & DISPLACEMENT $=$ & $0.103770518453790+01$ \\
\hline
\end{tabular}




\begin{tabular}{|c|c|c|c|}
\hline$M E=$ & & ISPLACEMENT $=$ & \\
\hline & 2.270 & I SPLACEMENT $=$ & $0.10470999932314 D+01$ \\
\hline $1 E=$ & & ISPLACEMENT $=$ & $0.105183280291710+01$ \\
\hline ME & .290 , & DI SPLACEMENT & $0.10565913655695 \mathrm{D}+01$ \\
\hline$M E=$ & 2.300, & I SPLACEMENT $=$ & $0.106137849050690+01$ \\
\hline ME $=$ & .310, & I SPLACEMENT $=$ & $0.11476631005725 \mathrm{D}+01$ \\
\hline ME $=$ & & ISPLACEMENT $=$ & $0.115331856957670+01$ \\
\hline ME $=$ & & I SPLACEMENT $=$ & $0.11590500263745 D+01$ \\
\hline ME & & ISPLACEMENT $=$ & $0.116486318019100+01$ \\
\hline ME $=$ & & I SPLACEMENT $=$ & $0.117076216046980+01$ \\
\hline ME $=$ & 360, & ISPLACEMENT $=$ & $0.117675169747580+01$ \\
\hline ME $=$ & & DISPLACEMENT $=$ & $0.118283658117380+01$ \\
\hline ME & & ISPLACEMENT = & $0.118902106815600+01$ \\
\hline ME $=$ & 90, & ISPLACEMENT $=$ & $0.119531125209590+01$ \\
\hline IME $=$ & 00 & ISPLACEMENT $=$ & $900+01$ \\
\hline ME $=$ & & DISPLACEMENT $=$ & 0.1208226 \\
\hline ME & & DISPLACEMENT $=$ & $640+01$ \\
\hline IME $=$ & & I SPLACEMENT $=$ & $190+01$ \\
\hline ME $=$ & & DISPLACEMENT $=$ & $0.12285147870224 \mathrm{D}+01$ \\
\hline IME & & DISPLACEMENT $=$ & 0.1235542 \\
\hline ME & & D I SPLACEMENT & 0.12427109 \\
\hline ME $=$ & & ISPLACEMENT $=$ & $0.125002699252970+01$ \\
\hline ME $=$ & & I SPLACEMENT $=$ & $0.125749538447970+01$ \\
\hline ME $=$ & & DISPLACEMENT $=$ & $0.12651217887352 D+01$ \\
\hline ME & & DI SPLACEMENT & 0.12729111 \\
\hline ME $=$ & .510, & ISPLACEMENT $=$ & $0.12808706969625 \mathrm{D}+01$ \\
\hline ME $=$ & & ISPLACEMENT $=$ & $0.128900555539860+01$ \\
\hline ME $=$ & & DISPLACEMENT $=$ & $0.12973216346622 \mathrm{D}+01$ \\
\hline ME $=$ & & DISPLACEMENT $=$ & $0.130582410754550+01$ \\
\hline$M E=$ & & DISPLACEMENT & $0.131452066497270+01$ \\
\hline MF & & DI SPLACEMENT & $0.132341665146820+01$ \\
\hline
\end{tabular}




\section{Appendix B}

Another sample program - use physical units rather nondimensionalized numbers. 

PROGRAM GPLATE

DOUBLE PRECISION DISPV(1024)

DIMENSION TIME(1024)

INTEGER YORNO

WRITE $(1, *)$ 'THIS PROGRAM WILL COMPUTE THE DISPLACEMENT U SUB I AT' WRITE $(1, *)$ ' A POINT ON THE SURFACE OF AN INFINITE PLATE DUE TO A' WRITE $(1, *)$ ' POINT STEP IMPULSIVE FORCE $F$ SUB $J$ OR A POINT LINEAR' WRITE $(1, *)$ ' RAMP FORCE DIPOLE F SUB $J, K$ ACTING AT ANOTHER POINT' WRITE(1,*)' ON THE SURFACE OF THE PLATE.' WRITE $(1, *)$ ' INPUTS REQUIRED ARE PROMPTED AND TYPED IN ON THE ' WRITE $(1, \star)$ ' XEYBOARD.'

WRITE(1,*)' '

10 WRITE $(1, *)$ ' SUBSCRIPTS OF $G=$ ? (i. e. $11,13,131,322$, etc.)'

$\operatorname{READ}(1, *)$ INDEX

WRITE $(1, *)$ ' SHEAR WAVE SPEED = ?'

READ $(1, *)$ SHSPD

WRITE $(1, *)$ ' LONGITUDINAL WAVE SPEED = ?'

$R E A D(1, *)$ PSPD

100 WRITE $(1, *)$ 'ARE SOURCE AND DETECTER ON THE SAME SIDE OF THE PLATE' WRITE(1,*)' PLEASE TYPE 1 FOR YES'

$\operatorname{READ}(1, \star)$ YORNO

IF (YORNO.EQ.1) THEN

$2 D=0.5$

ELSE

$Z D=-0.5$

END IF

WRITE $(1, \star)$ ' WHAT IS THE THICKNESS OF THE PLATE ?'

$\operatorname{READ}(1, \star)$ HTHICK

WRITE $(1, *)$ ' WHAT IS THE DISTANCE BETWEEN DETECTER AND SOURCE'

WRITE $(1, *)$ ' OR EPICENTER ?'

$\operatorname{READ}(1, *) X$

WRITE $(1, \star)$ ' SAMPLING TIME INTERVAL = ?'

$\operatorname{READ}(1, *)$ DT

WRITE $(1, *)$ ' NUMBER OF SAMPLING POINTS $=$ ? $(\operatorname{MAX}=1024)$ '

$\operatorname{READ}(1, *)$ NPT

WRITE $(1, \star)$ ' SHEAR MODULUS OF THE PLATE = ?'

$\operatorname{READ}(1, *)$ SHMDUL

WRITE $(1, *)$ ' THE OUTPUT WILL BE TIME VERSUS DISPLACEMENT.'

WRITE $(1, *)+$ ।

c

ALPHA $=$ SHSPD/PSPD

$X D=X / H T H I C K$

TSCALE $=$ HTHICK/SHSPD

DELTAT $=$ DT/TSCALE

DSCALE $=1 . /\left(2 .{ }^{\star} A \operatorname{SIN}(1.0){ }^{\star}\right.$ SHMDUL*HTHICK $)$

C

WRITE $(2, *) 1 \quad 1$

WRITE $(2, *)$ ' THE FOLLOWINGS ARE INPUT PARAMETERS:'

$\operatorname{WRITE}(2, *)$

INDEX $=1$, INDEX

$\operatorname{WRITE}(2, *)$

SHEAR WAVE SPEED $=1$, SHSPD

WRITE $(2, *)$ 'LONGITUDINAL WAVE SPEED $=1$,PSPD

WRITE $(2, *)$ 'THICXNESS OF THE PLATE $=1$, HTHICK

WRITE $(2, *)$ 'SAMPLING TIME INTERVAL $=1$, DT 


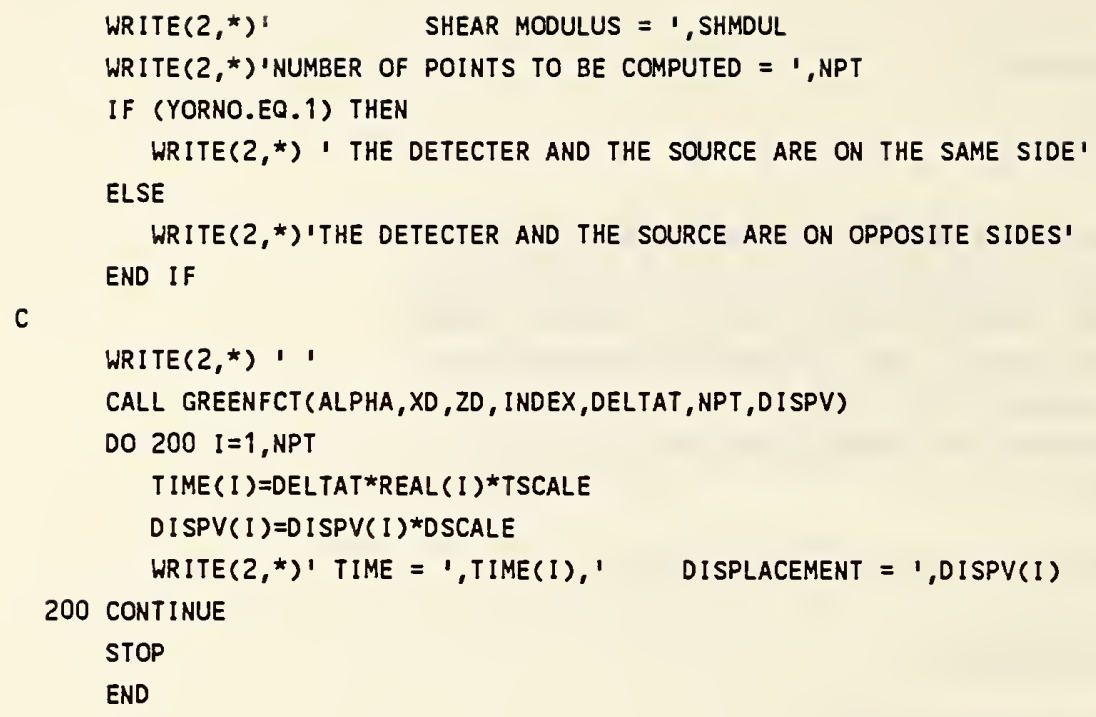




\section{Appendix C}

Listing of Subroutines for Computing Green's function of an infinite plate. 


\begin{tabular}{|c|c|c|}
\hline & : & GR000010 \\
\hline c & & GR000020 \\
\hline & SUBROUTINE GREENFCT (ALPHA, XD, ZD, INDEX, TDELTA, NPOINT, DISPL) & GR000030 \\
\hline C & & GR000040 \\
\hline & 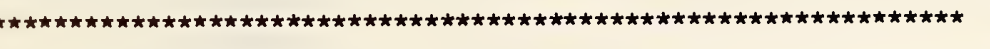 & GR000050 \\
\hline C & & GR000060 \\
\hline C & TO COMPUT GREEN'S FUNCTION - DISPLACEMENT AS A FUNCTION OF TIME & GR000070 \\
\hline C & - FOR GIVEN TEST CONFIGURATION & GR000080 \\
\hline 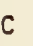 & & GR000090 \\
\hline : & INPUTS : & GR000100 \\
\hline c & ALPHA = SHEAR WAVE SPEED / LONGITUDINAL WAVE SPEED & GR000110 \\
\hline C & $X D=X$ COORDINATE OF THE DETECTOR & GR000120 \\
\hline c & $Z D=Z$ COORDINATE OF DETECTOR & GR000130 \\
\hline c & $Z D=0.5 \Rightarrow$ DETECTOR \& SOURCE ON THE SAME SIDE & GR000140 \\
\hline C & $Z D=-0.5 \Rightarrow$ DETECTOR \& SOURCE ON OPPOSIT SIDES & GR000150 \\
\hline c & INDEX = SUBSCRIPT OF GREEN'S FUNCTION, i.e. 33,322 & GR000160 \\
\hline c & TDELTA = TIME INCREMENT & GR000170 \\
\hline c & NPOINT = TOTAL NUMBER OF POINTS TO BE COMPUTED & GR000180 \\
\hline C & & GR000190 \\
\hline C & OUTPUT: & GR000200 \\
\hline C & 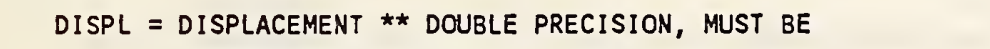 & GR000210 \\
\hline c & DIMENSIONED (NPOINT) & GR000220 \\
\hline c. & n. & GR000230 \\
\hline C & & GR000240 \\
\hline C & NOTE 1: & GR000250 \\
\hline C & ALL INPUTS AND OUTPUTS ARE NONDIMENSIONALIZED PARAMETERS & GR000260 \\
\hline C & DISPL $=$ NORMALIZED DISPLACEMENT $=$ ACTUAL DISPLACEMENT * PI & GR000270 \\
\hline C & * SHEAR MODULUS * PLATE THICKNESS / FORCE & GR000280 \\
\hline C & $T=$ NORMALIZED TIME = ACTUAL TIME *SHEAR WAVE SPEED / PLATE & GR000290 \\
\hline C & THICKNESS & GR000300 \\
\hline C & DISPL MUST BE DIMENSIONED IN THE CALLING PROGRAM (NPOINT) & GR000310 \\
\hline C & & GR000320 \\
\hline c- & 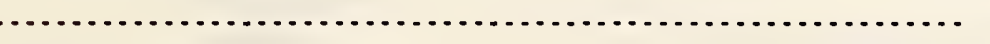 & GR000330 \\
\hline C & & GR000340 \\
\hline C & NOTE 2: & GR000350 \\
\hline C & THE ARRIVAL TIMES ARE STORED IN RAYTIME(N,I) & GR000360 \\
\hline C & $N=$ THE NTh RAY & GR000370 \\
\hline C & $\operatorname{RAYTIME}(N, 3)=$ THE ARRIVAL TIME & GR000380 \\
\hline C & $\operatorname{RAYTIME}(N, 1)=N 1$, NUMBER OF P TRIPS & GR000390 \\
\hline C & $\operatorname{RAYTIME}(N, 2)=N 2$, NUMBER OF S TRIPS & GR000400 \\
\hline C & IF N2<0, IT IS A HEAD WAVE & GR000410 \\
\hline C & IF $N 1=-1$, IT IS SURFACE WAVE & GR000420 \\
\hline C & IF $N 1=0$ AND N2=0, IT IS A RAYLEIGH WAVE & GR000430 \\
\hline C & & GR000440 \\
\hline C & THE TIME OF ARRIVAL IMFORMATION IS ALSO PRINTED ON LU 6 & GR000450 \\
\hline C & & GR000460 \\
\hline c- & 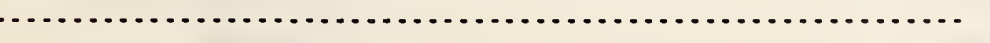 & GR000470 \\
\hline C & & GR000480 \\
\hline & DOUBLE PRECISION DISPL(NPOINT) & GR000490 \\
\hline & DIMENSION TA(101), CN $(101,3), \operatorname{RAYTIME}(101,3)$ & GR000500 \\
\hline C & NRAY = NUMBER OF MAXIMUM RAYS EXPECTED & GR000510 \\
\hline & DATA NRAY/101/ & GR000520 \\
\hline C & & GR000530 \\
\hline & CALL INIT(ALPHA, XD, ZD, INDEX) & GR000540 \\
\hline
\end{tabular}


CALL TIMEARRI (ALPHA, XD, ZD, NRAY, RAYTIME, TA, CN)

GR000550

WRITE $\left(6,{ }^{*}\right)$ ' I N1 N2 TIME ARRIVAL'

DO $500 I=1$, NRAY

WRITE $(6,499) I, \operatorname{RAYTIME}(1,1), \operatorname{RAYTIME}(1,2), \operatorname{RAYTIME}(1,3) \quad \operatorname{GRO00580}$

499 FORMAT (2X, I5, 3X, 3F 10.5)

500 CONTINUE

DO $1000 \quad I=1$, NPOINT

TIMEI $=$ FLOAT $(1) * T D E L T A$

GR000570

GR000590

GR000600

GR000610

GR000620

CALL GREENSUB (ALPHA, INDEX, XD , ZD, NRAY, TIMEI , RAYTIME,DISPL(I))

GR000630

WRITE $\left(6,{ }^{*}\right) ' T I M E=1, T I M E I, ' \quad$ DISPL $=1, D I S P L(I)$

1000 CONTINUE

GR000640

GR000650

RETURN

GR000660

END

GR000670

GR000680

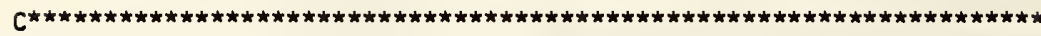

GR000690

SUBROUT INE INIT (ALPHA, XD, ZD, INDEX)

GR000700

C

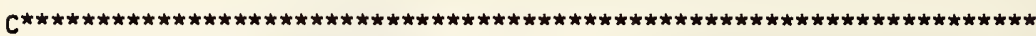

IMPLICIT DOUBLE COMPLEX (Y)

GR000710

GR000720

GR000730

IMPLICIT DOUBLE PRECISION (D)

GR000740

COMMON /BLKO/DXD,DT,DZ,DA

GR000750

COMMON /BLK1/KASE,M,L,K,N1,N2,D, DTHETA

COMMON /BLK3/YI, YONE, YTWO, YTHREE, YFOUR, YEIGHT, YZERO

GR000760

COMMON /BLK5/YR, Y1, Y2

GR000770

$D X D=D B L E(X D)$

$D Z=D B L E(Z D)$

$D A=$ CBLE(ALPHA)

IF (ZD.EO.0.5) KASE $=1$

IF (ZD.EO.-0.5) KASE $=2$

IF (INDEX.LT.100) THEN

$K=0$

$M=I N D E X / 10$

$L=I N D E X \cdot M * 10$

ELSE IF ((INDEX.GT.100).AND.(INDEX.LT.334)) THEN

$M=I N D E X / 100$

$L=\left(\right.$ INDEX $\left.\cdot M^{*} 100\right) / 10$

$K=\left(\right.$ INDEX $\left.\cdot M^{*} 100 \cdot L^{*} 10\right)$

ELSE

WRITE $\left(6,{ }^{*}\right)$ ' WRONG INDEX '

PAUSE

END IF

$Y I=D C M P L X(0 . D 0,1 . D 0)$

YONE $=D C M P L X(1 . D 0,0 . D 0)$

$Y T W O=D C M P L X(2 . D O, 0 . D O)$

$Y T H R E E=D C M P L X(3 . D 0,0 . D 0)$

GR000780

GR000790

GR000800

GRO00810

GR000820

GR000830

GR000840

GR000850

GR000860

GR000870

GR000880

GR000890

GR000900

GR000910

GR000920

GR000930

GR000940

GR000950

GR000960

GR000970

GR000980

GR000990

GR001000

GR001010

GR001020

GR001030

GR001040

GR001050

GR001060

GR001070

GR001080

SUBROUT INE GREENSUB (ALPHA, INDEX, XD, ZD, NRAY, T, RAYTIME, DISPL)

GR001090 


\begin{tabular}{|c|c|c|}
\hline \multicolumn{2}{|r|}{ C } & GR001100 \\
\hline c & \multirow[t]{2}{*}{ SUBROUTINE TO COMPUTE THE GREENS FUNCTION OF A PLATE AT T } & GR001110 \\
\hline c & & GR001120 \\
\hline C & INPUTS: & GR001130 \\
\hline c & CONFIGURATION PARAMETERS: & GR001140 \\
\hline C & DETECTOR LOCATION: & GR001150 \\
\hline C & $X=X D$ & GR001160 \\
\hline c & $Y=0$ & GR001170 \\
\hline c & $Z=Z D, Z D=0.5 \Rightarrow$ TOP; $Z D=-0.5 \Rightarrow$ BOTTOM & GR001180 \\
\hline c & SOURCE LOCATION: & GR001190 \\
\hline c & $x_{0}=0$ & GR001200 \\
\hline c & $Y 0=0$ & GR001210 \\
\hline c & \multirow[t]{2}{*}{$Z O=+0.5$ (ON TOP OF THE PLATE) } & GR001220 \\
\hline c & & GR001230 \\
\hline C & MATERIALS PARAMETERS: & GR001240 \\
\hline C & \multirow[t]{2}{*}{ ALPHA = RATIO OF SHEAR VELOCITY TO LONGITUDINAL VELOCITY } & GR001250 \\
\hline C & & GR001260 \\
\hline c & OTHER PARAMETERS: & GR001270 \\
\hline C & INDEX: SUBSCRIPTS OF GREENS FUNCTION i.e. 33 , or 113 & GR001280 \\
\hline c & NRAY: MAXIMUM NUMBER OF RAYS EXPECTED. $\sim 100$ & GR001290 \\
\hline c & \multirow[t]{2}{*}{ RAYTIME: TIME OF ARRIVAL TABLE OF DIMENSION (NRAY,3) } & GR001300 \\
\hline c & & GR001310 \\
\hline C & \multirow{3}{*}{$\begin{array}{c}T=\text { NORMALIZED TIME }=\text { ACTUAL TIME *SHEAR WAVE SPEED / PLATE } \\
\text { THICKNESS }\end{array}$} & GR001320 \\
\hline c & & GR001330 \\
\hline c & & GR001340 \\
\hline c & \multirow[t]{2}{*}{ DISPLACEMENT WILL BE CORRECT ONLY IF T < RAYTIME(NRAY,3) } & GR001350 \\
\hline c & & GR001360 \\
\hline C & OUTPUT: & GR001370 \\
\hline C & DISPL $=$ NORMALIZED DISPLACEMENT $=$ ACTUAL DISPLACEMENT * PI & GR001380 \\
\hline c & * SHEAR MODULUS * PLATE THICKNESS / FORCE & GR001390 \\
\hline C & & GR001400 \\
\hline C & NOTE: SUBROUTINE INIT(ALPHA, XD, ZD, INDEX) AND & GR001410 \\
\hline C & SUBROUTINE TIMEARRI $(\ldots \ldots$.$) SHOULD BE CALLED FIRST IN$ & GR001420 \\
\hline C & THE MAIN PROGRAM. & GR001430 \\
\hline C & & GR001440 \\
\hline & IMPLICIT DOUBLE COMPLEX (Y) & GR001450 \\
\hline & IMPLICIT DOUBLE PRECISION (D) & GR001460 \\
\hline & DOUBLE PRECISION DINTG & GR001470 \\
\hline & DIMENSION RAYTIME(NRAY,3) & GR001480 \\
\hline & COMMON /BLKO/DXD,DT,DZ,DA & GR001490 \\
\hline & COMMON /BLK1/KASE,M,L,K,N1,N2,D,DTHETA & GR001500 \\
\hline & CCMMON /BLK2/YD, Y, YP, YQ, YRO, YRP, YRM, YDELTA, YPSQP1, YQSQP1, & GR001510 \\
\hline & 1 YPSOM1, YQSQM1, YSQRP1, YSQRQ1, YY, YA, YAA, YETA1, YETA2 & GR001520 \\
\hline & 2 ,YDPHI & GR001530 \\
\hline & COMMON /BLK3/YY, YONE, YTWO, YTHREE, YFOUR, YEIGHT, YZERO & GR001540 \\
\hline & COMMON /BLK5/YR, Y1, Y2 & GR001550 \\
\hline C & & GR001560 \\
\hline & EXTERNAL DINTG & GR001570 \\
\hline C & & GR001580 \\
\hline c & & GR001590 \\
\hline & IF ( (INDEX.EQ.12).OR. (INDEX.EQ.21).OR. & GR001600 \\
\hline & $+\quad($ INDEX.EQ.23).OR.(INDEX.EQ.32).OR. & GR001610 \\
\hline & (INDEX.EQ.121).OR.(INDEX.EQ.211).OR. & GR001620 \\
\hline & (INDEX.EQ.231).OR.(INDEX.EQ.321).OR. & GR001630 \\
\hline & $+\quad$ (INDEX.EQ.123).OR. (INDEX.EQ.213).OR. & GR001640 \\
\hline
\end{tabular}


$+\quad$ (INDEX.EQ.233).OR.(INDEX.EQ.323).OR .

GR001650

$+\quad$ (INDEX.EQ.132).OR.(INDEX.EQ.312).OR.

GR001660

$+\quad$ (INDEX.EQ.112).OR.(INDEX.EQ.222).OR.(INDEX.EQ.332)) THEN

GR001670 DISPL $=0.000$

RETURN

GR001680

ELSE

C

$D Z E R O=0 . D O$

GR001690

GR001700

GR001710

GR001720

C NMAX = NRAY; MaXimum number of rays. The same as DIM RAYTIME

GR001730

NMAX $=$ NRAY

IF(T.GT. (RAYTIME(NRAY, 3))) THEN

GR001740

GR001750

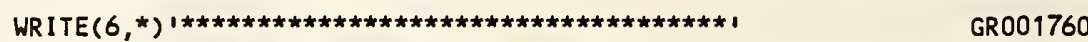

WRITE $(6, *)$ 'ERROR IN SUBROUTINE GREEN'

WRITE $(6, *)$ 'T > TARRIVAL (NRAY), INCREASE NRAY.'

GR001770

GR001780

WRITE $\left(6,{ }^{*}\right)$ 'PROGRAM IS PAUSED'

GR001790

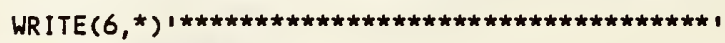

PAUSE 'GREENSUB '

GR001800

GR001810

ENDIF

GR001820

GR001830

$D T=D B L E(T)$

GR001840

GR001850

GR001860

DSURF $=0 . D 0$

IF (KASE.EQ.1) THEN

CALL SFWAVE (ALPHA, XD, T, INDEX, DSURF)

GR001870

GR001880

GR001890

GR001900

GR001910

GR001920

GR001930

GR001940

GR001950

GR001960

GR001970

GR001980

GR001990

GR002000

GR002010

GR002020

GR002030

GR002040

GR002050

GR002060

GR002070

GR002080

GR002090

GR002100

GR002110

GRO02120

GR002130

GR002140

GR002150

GR002160

GR002170

GR002180

C8000 $Y=Y Z E R O$; set trY root eq. 0

$8000 \quad Y=Y Z E R O$

GR002190 
RETURN

GR002220

END IF

GR002230

END

GR002240

GR002250

GR002260

DOUBLE PRECISION FUNCTION DINTG(DINIT,DEND)

GR002270

GR002280

C

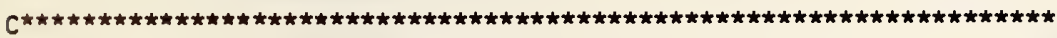

GR002290

GR002300

IMPLICIT DOUBLE PRECISION (D)

GR002310

GR002320

GR002330

DOUBLE PRECISION EPS, $R, E, W(50,6)$, FMIN, FMAX, $F$

GR002340

INTEGER NINT, NMAX, KF, I FLAG

GR002350

LOGICAL RST

GR002360

GR002370

GR002380

EXTERNAL $F$

GR002390

GR002400

$E P S=1.0 .6$

RST $=$.FALSE .

GR002410

GR002420

NMAX $=50$

GR002430

NINT $=1$

GR002440

$W(1,1)=$ DINIT

GR002450

$W(2,1)=$ DEND

GR002460

GR002470

GR002480

CALL DGLQ1 (F, DINIT , DEND , EPS, R, E, NINT , RST, W, NMAX, FMIN , FMAX, KF

GR002490

GR002500

GR002510

DINTG $=R$

GR002520

RETURN

GR002530

END

GR002540

GR002550

GR002560

GR002570

DOUBLE PRECISION FUNCTION F(DS)

GR002580

GR002590

GR002600

GR002610

IMPLICIT DOUBLE COMPLEX (Y)

GR002620

GR002630

GR002640

GR002650

GR002660

GR002670

GR002680

GR002690

GR002700

GR002710

GR002720

GR002730

DK2 $=$ DBLE (REAL (N2))

GR002740 
c

CALL PHIEQO(DK1,DK2,DA,DT,DXD,DS, Y,DERR, NROOT)

C WRITE $\left(6,{ }^{\star}\right) D K 1, D K 2, D S, Y, D E R R$, NROOT

IF (NROOT.EQ.0) THEN

$F=0 . D 0$

C

\section{ELSE}

CALL COMMON(DS)

CALL RSTAR(YRSTAR, IDUMMY)

CALL QD (YQD, IDUMMY)

CALL QSU(YQS, IDUMMY)

C

YINTG $=$ YZERO

DO $200 \quad I=1,2$

DO $100 \mathrm{~J}=1,2$

$Y I N T G=Y I N T G+Y Q D(M, I) \star Y R S T A R(I, J) \star Y O S(L, J)$

100 CONTINUE

200 CONTINUE

C

IF ((N1.EQ.0).AND. (N2.GT.0))

$1 Y$ INTG $=Y I N T G+Y O D(M, 3) \star Y O S(L, 3)$

c

$Y I N T G=Y I N T G * Y D P H I / C D S Q R T\left(D C M P L X\left(D X D \star D X D-D S^{\star} D S\right)\right)$

$F=D B L E(Y I N T G)$

c

END IF

RETURN

END

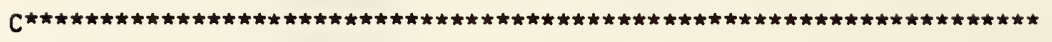

C

SUBROUTINE PHIEQO(DK1,DK2,DA,DT, DXD,D, YROOT, DERR, NROOT)

C

C

C

C GIVEN DK1,DK2,DA,DT,D TO SOLVE PHI (YROOT $)=0$

IMPLICIT DOUBLE PRECISION (D)

IMPLICIT DOUBLE COMPLEX (Y)

DOUBLE PRECISION $T, R, A, C 1, C 2$

COMMON /PARM1/T, R, A, C1, C2

C

$Y O L D=Y R O O T$

IER $=0$

DYREAL $=0 . D 0$

YPH $I=D C M P L X(0 . D 0,0.00)$

$T=D T$

$R=D$

$A=D A$

$C 1=D K 1$

$C 2=D K 2$

EPS $=1 . E-12$

IEND $=100$

c

NROOT $=1$

IF (DK2.EQ.0.DO) THEN
GR002750

GR002760

GR002770

GR002780

GR002790

GR002800

GR002810

GR002820

GR002830

GR002840

GR002850

GR002860

GR002870

GR002880

GR002890

GR002900

GR002910

GR002920

GR002930

GR002940

GR002950

GR002960

GR002970

GR002980

GR002990

GR003000

GR003010

GR003020

GR003030

GR003040

GR003050

GR003060

GR003070

GR003080

GR003090

GR003100

GR003110

GR003120

GR003130

GR003140

GR003150

GR003160

GR003170

GR003180

GR003190

GR003200

GR003210

GR003220

GR003230

GR003240

GR003250

GR003260

GR003270

GR003280

GR003290 


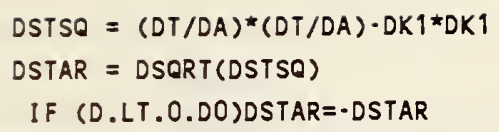




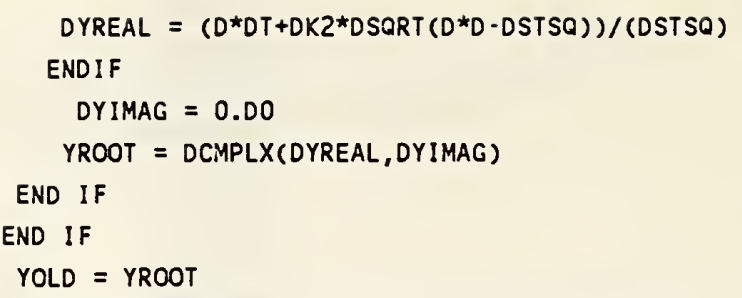

C CALL QUADRTIC EQ. TO FIND $Y \star \star 2$

GR003850

GR003860

GR003870

GR003880

GR003890

GR003900

GR003910

GR003920

GR003930

GR003940

GR003950

GR003960

GR003970

GR003980

GR003990

GR004000

GR004010

GR004020

GR004030

GR004040

GR004050

GR004060

GR004070

GR004080

GR004090

GR004100

GR004110

GR004120

GR004130

GR004140

GR004150

GR004160

GROO4170

GR004180

GR004 190

GR004200

GR004210

GR004220

GR004230

GR004240

GR004250

GR004260

GR004270

GR004280

GR004290

GR004300

GR004310

GR004320

GR004330

GR004340

GR004350

GR004360

GR004370

GR004380

GR004390 
CALL QUADRT (B, DROOT1, DROOT2, TOL, NOROOT)

GR004400

C

Y $1=$ CDSORT (DCMPLX(DROOT 1 (1), DROOT 1 (2)))

GR004410

$Y 2=\operatorname{CDSQRT}(D C M P L X(D R O O T 2(1), D R O O T 2(2)))$

GR004420

IF (DIMAG(Y1).GT.0.D0) Y $Y=-Y 1$

IF (DIMAG $(Y Z) . G T \cdot 0 . D 0) \quad Y Z=-Y Z$

GR004430

GR004440

$Y F 1=Y F(Y 1)$

$Y F 2=Y F(Y 2)$

DERR $1=\operatorname{CDABS}(Y F(Y 1))$

DERR2 $=\operatorname{CDABS}(Y F(Y 2))$

IF (DERR1.LT.DERR2) $Y=Y 1$

IF (DERR1.GE.DERR2) $Y=Y 2$

GR004450

GR004460

GR004470

GR004480

GR004490

GR004500

GR004510

GR004520

IF (DIMAG(Y).GE.0.DO) Y=DCONJG(Y)

GR004530

GR004540

GR004550

RETURN

GR004560

END

GR004570

GR004580

DOUBLE COMPLEX FUNCTION PHIO(Z)

GR004590

GR004600

C

GR004610

GR004620

FUNCTION PHIO

GR004630

GR004640

GR004650

DOUBLE PRECISION $T, R, A, C 1, C 2$

GR004660

DOUBLE COMPLEX $Z$, YAA, YONE

GR004670

COMMON /PARM $1 / T, R, A, C 1, C 2$

GR004680

$Y O N E=D C M P L X(1 . D 0,0 . D 0)$

GR004690

$Y A A=D C M P L X\left(A^{\star} A, 0 . D 0\right)$

GR004700

GR004710

$P H I O=D C M P L X(R, 0 . D O)-D C M P L X(T, 0 . D O) \star Z$

GR004720

$1-D C M P L X(C 1,0 . D 0) * C D S O R T(Y A A \star Z \star Z-Y O N E)$

2 -DCMPLX $(C 2,0 . D 0) \star C D S O R T(Z \star Z$-YONE)

C

RETURN

GR004730

GR004740

GR004750

GR004760

GR004770

GR004780

GR004790

GR004800

GR004810

GR004820

GR004830

GR004840

GR004850

GR004860

GR004870

DOUBLE PRECISION $T, R, A, C 1, C 2$

GR004880

GR004890

GR004900

GR004910

GR004920

GR004930

GR004940 
$1-D C M P L X(C 1,0 . D 0) * Y A A * Z / C D S Q R T\left(Y A{ }^{*} Z * Z-Y O N E\right) \quad$ GR004950

$2 \cdot \operatorname{DCMPLX}(C 2,0 . D 0) * Z / C D S Q R T(Z \star Z-Y O N E) \quad$ GR004960

C

RETURN

GR004970

END

GR004980

GR004990

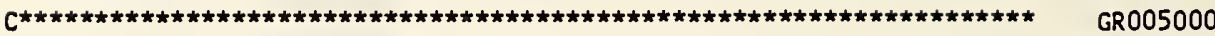

C

SUBROUTINE COMMON(DS)

GR005010

GR005020

C

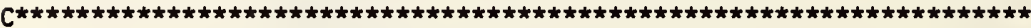

GR005030

GR005040

GR005050

GR005060

GR005070

GR005080

GR005090

IMPLICIT DOUBLE COMPLEX (Y)

GR005 100

DOUBLE PRECISION ETA1,ETA2SQ,DD,AA

COMMON /BLKO/DXD,DT,DZ,DA

GR005110

GR005120

GR005130

GR005 140

COMMON /BLK2/YD, Y, YP, YQ, YRO, YRP, YRM, YDELTA, YPSOP 1 , YQSQP 1 ,

GR005150

GR005 160

GR005170

GR005180

GR005190

GR005200

GR005210

GR005220

GR005230

GR005240

GR005250

GR005260

GR005270

GRO05280

GR005290

GR005300

GR005310

GR005320

GR005330

GR005340

GR005350

GR005360

GR005370

GR005380

GR005390

GR005400

GR005410

GR005420

GR005430

GR005440

GR005450

GR005460

GR005470

GR005480

GR005490 
$Y M=(Y Q S O M 1 * Y Q S Q M 1-Y F O U R * Y P * Y Q)$

GR005500

$Y R O=Y M / Y D E L T A$

$Y R P=-(Y F O U R \star Y P \star Y Q S O M 1) /(Y A * Y D E L T A)$

GR005510

GR005520

$Y R M=(Y R O \star Y R O-Y O N E) / Y R P$

GR005530

C

$Y D P H I=Y O N E /(Y D+(D C M P L X(D N 1,0 . D O) / Y P)+(D C M P L X(D N 2,0 . D 0) / Y Q))$

GR005540

RETURN

GR005550

GR005560

END

GR005570

GR005580

GR005590

SUBROUTINE RSTAR (YRSTAR, IDUMMY)

GR005600

GR005610

GR005620

GR005630

GR005640

GR005650

GR005660

IMPLICIT DOUBLE COMPLEX (Y)

GR005670

GR005680

DIMENSION YRSTAR(3,3), XSUM(20)

GR005690

COMMON /BLKO/DXD,DT,DZ,DA

COMMON /BLK1/KASE, $M, L, X, N 1, N 2, D$, DTHETA

COMMON /BLK2/YD, Y, YP, YO, YRO, YRP, YRM, YDELTA, YPSOP1, YOSOP1,

GR005700

GR005710

1 YPSOM1, YOSOM1, YSORP1, YSORO1, YY, YA, YAA, YETA1, YETA2

2 ,YDPHI

COMMON /BLK3/YI, YONE, YTWO, YTHREE, YFOUR, YEIGHT, YZERO

C

EXTERNAL KDELTA

GR005720

GR005730

GR005740

GR005750

GR005760

GR005770

GR005780

GR005790

IDUMMY $=0$

GR005800

$\operatorname{YRSTAR}(3,3)=$ YONE

GR005810

$\operatorname{YRSTAR}(1,3)=\operatorname{YZERO}$

GR005820

$\operatorname{YRSTAR}(2,3)=Y Z E R O$

GR005830

$\operatorname{YRSTAR}(3,1)=\operatorname{YZERO}$

GR005840

GR005850

DO $2000 \mathrm{JS}=1,2$

GR005860

DO $1000 \mathrm{~J} 2=1,2$

GR005870

GR005880

GR005890

IF (J2.EO.2) $\mathrm{N2}=\mathrm{N2}-1$

GR005900

ISIGN $=(-1) \star \star(J 2 *(N 1+N 2)+N 2)$

GR005910

CALL COEFF (N1, N2, JS, J2, KD, KU, XL, KSUM)

GR005920

GR005930

GR005940

GR005950

GR005960

GR005970

GR005980

GR005990

GR006000

GR006010

GR006020

GR006030

GR006040 
END

GR006110

GR006120

C

GR006130

C

SUBROUTINE QD(YOD, IDUMMY)

GR006140

GR006150

$c$

GR006160

C

GR006170

C

C TO COMPUTE QD(I,J) BASED ON EQ. 6.21

GR006180

GR006190

c

IMPLICIT DOUBLE COMPLEX (Y)

IMPLICIT DOUBLE PRECISION (D)

COMMON /BLKO/DXD,DT,DZ,DA

COMMON /BLK1/KASE, M, L, K, N1, N2,D, DTHETA

COMMON /BLK2/YD, Y, YP, YQ, YRO, YRP, YRM, YDELTA, YPSQP1, YQSQP1,

1 YPSQM1, YQSQM1, YSQRP 1 , YSQRQ1, YY, YA, YAA, YETA 1, YETA2

2 ,YDPHI

COMMON /BLK3/YI, YONE, YTWO, YTHREE, YFOUR, YEIGHT, YZERO

C

DIMENSION YOD $(3,3)$

C

IDUMMY $=0$

DO $100 \quad I=1,3$

DO $90 \mathrm{~J}=1,3$

$$
Y \operatorname{YCD}(I, J)=Y Z E R O
$$

GR006200

GR006210

GR006220

GR006230

GR006240

GR006250

GR006260

GR006270

GR006280

GR006290

GR006300

GR006310

GR006320

GR006330

GR006340

GR006350

GR006360

GR006370

GR006380

GR006390

GR006400

GR006410

GR006420

GR006430

GR006440

GR006450

GR006460

GR006470

GR006480

GR006490

GR006500

GR006510

GR006520

GR006530

GR006540

GR006550

GR006560

GR006570

GR006580

GR006590 
END IF

GR006600

RETURN

GR006610

END

GR006620

C

GR006630

C

INTEGER FUNCTION $\operatorname{KDELTA}(I, J)$

GR006640

GR006650

C

GR006660

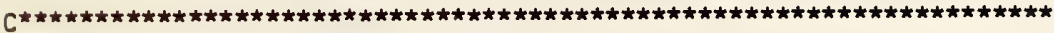

GR006670

C

IF (I.EQ.J) THEN

GR006680

KDELTA $=1$

GR006690

GR006700

ELSE

KDELTA $=0$

GR006710

GR006720

END IF

GR006730

RETURN

GR006740

END

GR006750

GR006760

GR006770

GR006780

INTEGER FUNCTION IBINO(M,N)

GR006790

GR006800

GR006810

IF (M.EQ.N)GO TO 11

IF $((M . L T . O) . O R .(N . L T . O))$ GO TO 10

GR006820

GR006830

GR006840

GR006850

GR006860

GR006870

GR006880

GR006890

GR006900

GR006910

GR006920

GR006930

GR006940

GR006950

GR006960

GR006970

GR006980

GR006990

GR007000

GR007010

GR007020

GR007030

GR007040

GR007050

GR007060

GR007070

GR007080

GR007090

GR007100

GR007110

GR007120

GR007130

GR007140

EXTERNAL KDELTA

$\operatorname{KD} 1=\operatorname{KDELTA}(0, N 1) \star \operatorname{KDELTA}(2, J 2)+\operatorname{KDELTA}(0, N 2) \approx K D E L T A(1, J 2)$

KD $1=K D 1 * K D E L T A(J S, J 2)$

$c$ 


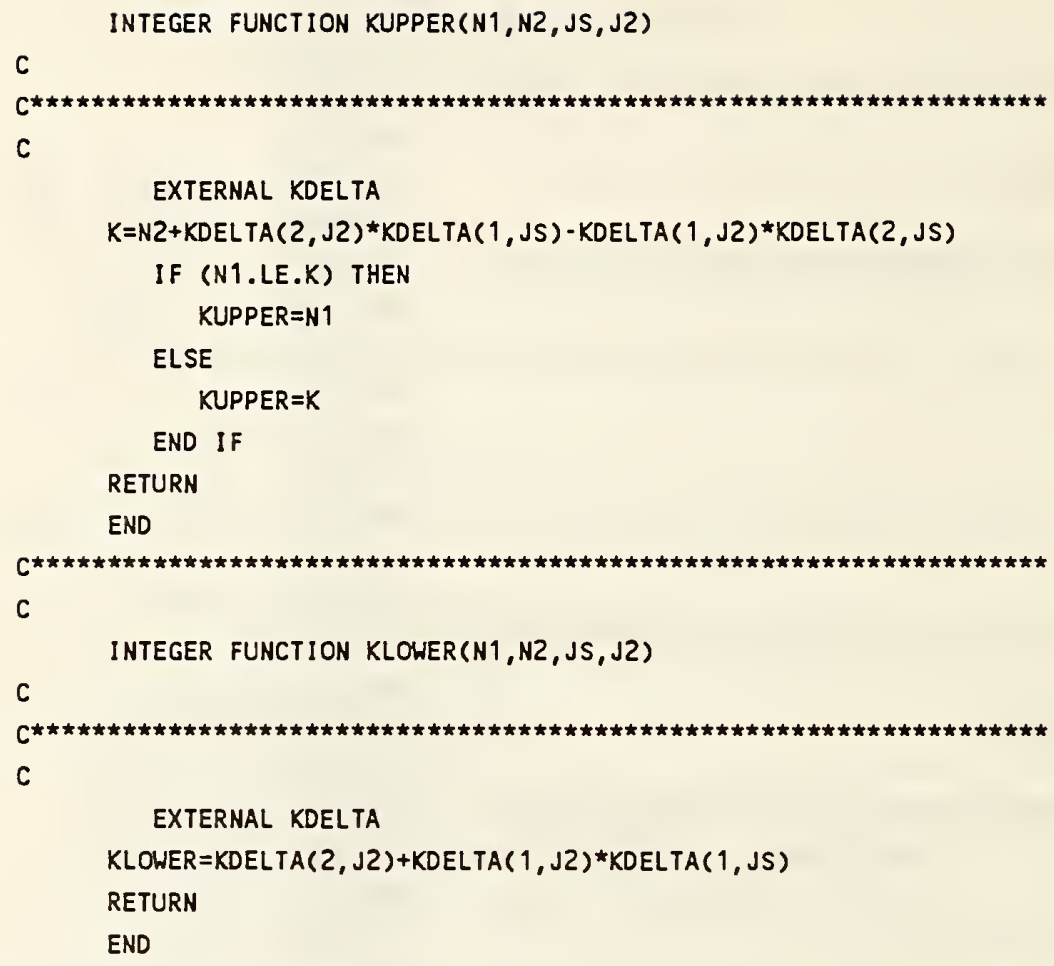

GR007150

GR007160

GR007170

GR007180

GR007190

GR007200

GR007210

GR007220

GR007230

GR007240

GR007250

GR007260

GR007270

GR007280

GR007290

GR007300

GR007310

GR007320

GR007330

GR007340

GR007350

GR007360

GR007370

GR007380

GR007390

GR007400

GR007410

GR007420

GR007430

GR007440

GR007450

GR007460

GR007470

GR007480

GR007490

GR007500

GR007510

GR007520

GR007530

GR007540

GR007550

GR007560

GR007570

GR007580

GR007590

GR007600

GR007610

GR007620

GR007630

GR007640

GR007650

GR007660

GR007670

GR007680

GR007690 
INTEGER FUNCTION KDELTA(I,J)

GR006630

GR006640

GR006650

C

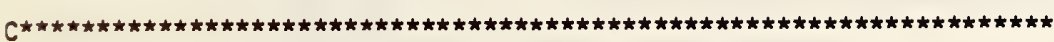

GR006660

C

If (I.EQ.J) THEN KDELTA $=1$

ELSE KDELTA $=0$

GR006670

GR006680

GR006690

GR006700

GR006710

GR006720

END IF

GR006730

GR006740

RETURN

GR006750

END

GR006760

GR006770

INTEGER FUNCTION IBINO(M,N)

GR006780

C

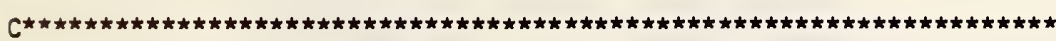

GR006790

GR006800

C

IF (M.EQ.N)GO TO 11

GR006810

GR006820

IF ((M.LT.O).OR.(N.LT.O)) GO TO 10

GR006830

$K=M \cdot N$

GR006840

If (K) $10,11,12$

GR006850

10 IBINO $=0$

GR006860

RETURN

GR006870

GR006880

GR006890

RETURN

GR006900

GR006910

GR006920

GR006930

GR006940

GR006950

GR006960

GR006970

GR006980

GR006990

GR007000

GR007010

GR007020

GR007030

GR007040

GR007050

GR007060

GR007070

GR007080

GR007090

GR007100

GR007110

GR007120

GR007130

GR007140

EXTERNAL KDELTA

$\mathrm{KD} 1=\mathrm{KD} 1 * \mathrm{KDEL} T A(\mathrm{JS}, \mathrm{J} 2)$

RETURN

END

$C$ 


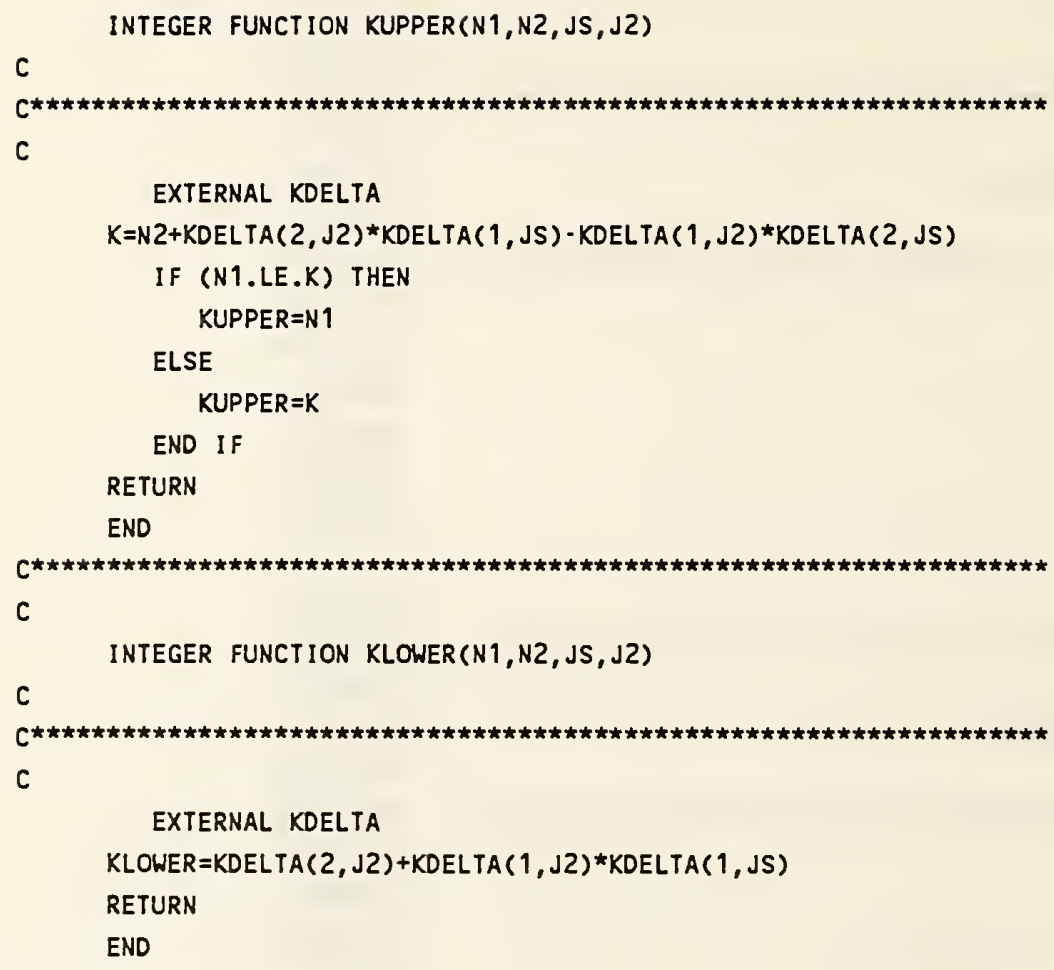

GR007150

GR007160

GR007170

GR007180

GR007190

GR007200

GR007210

GR007220

GR007230

GR007240

GR007250

GR007260

GR007270

GR007280

GR007290

GR007300

GR007310

GR007320

GR007330

GR007340

GR007350

GR007360

GR007370

GR007380

GR007390

GR007400

GR007410

GR007420

GR007430

GR007440

GR007450

GR007460

GR007470

GR007480

GR007490

GR007500

GR007510

GR007520

GR007530

GR007540

GR007550

GR007560

GR007570

GR007580

GR007590

GR007600

GR007610

GR007620

GR007630

GR007640

GR007650

GR007660

GR007670

GR007680

GR007690 


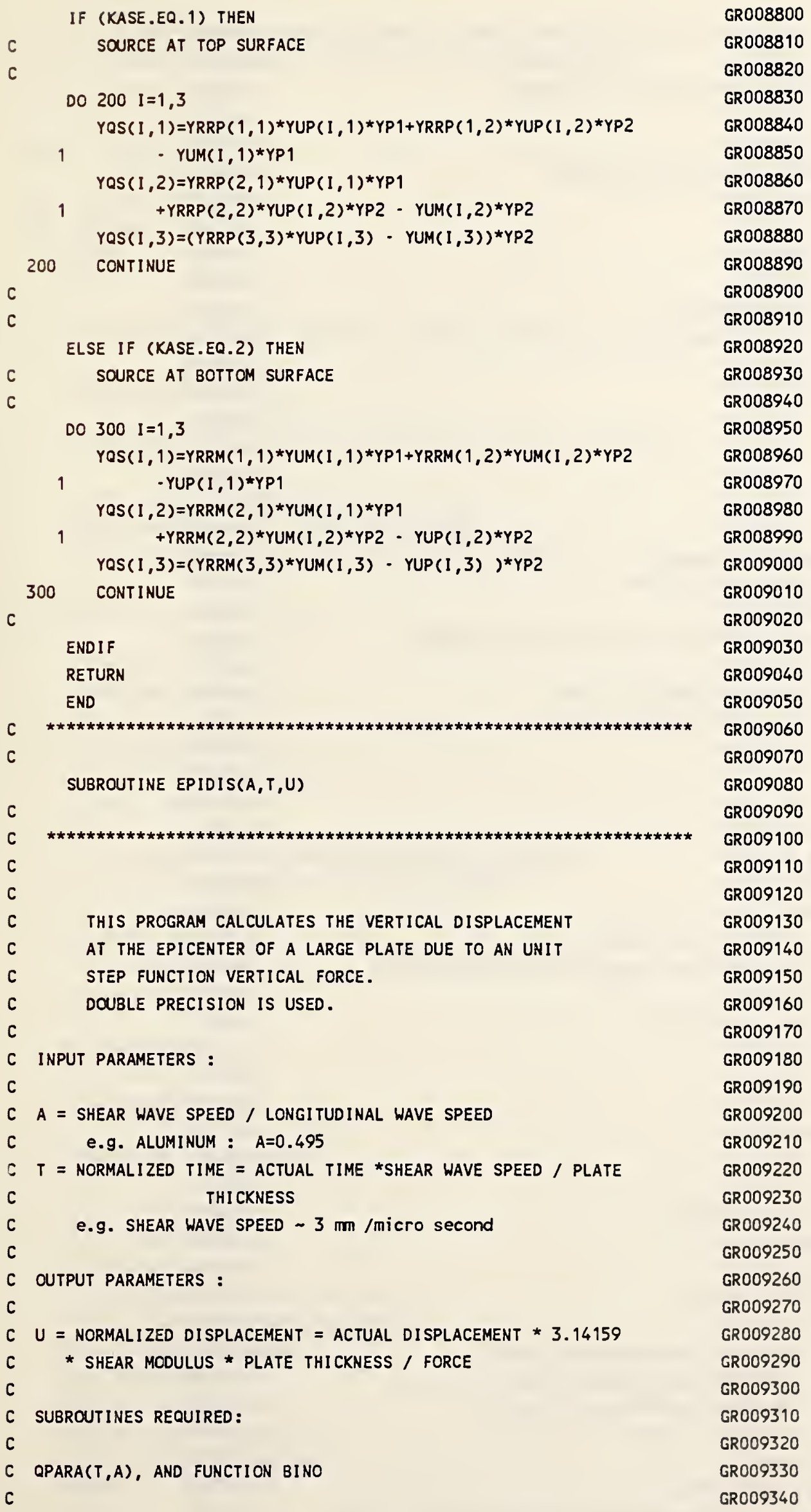




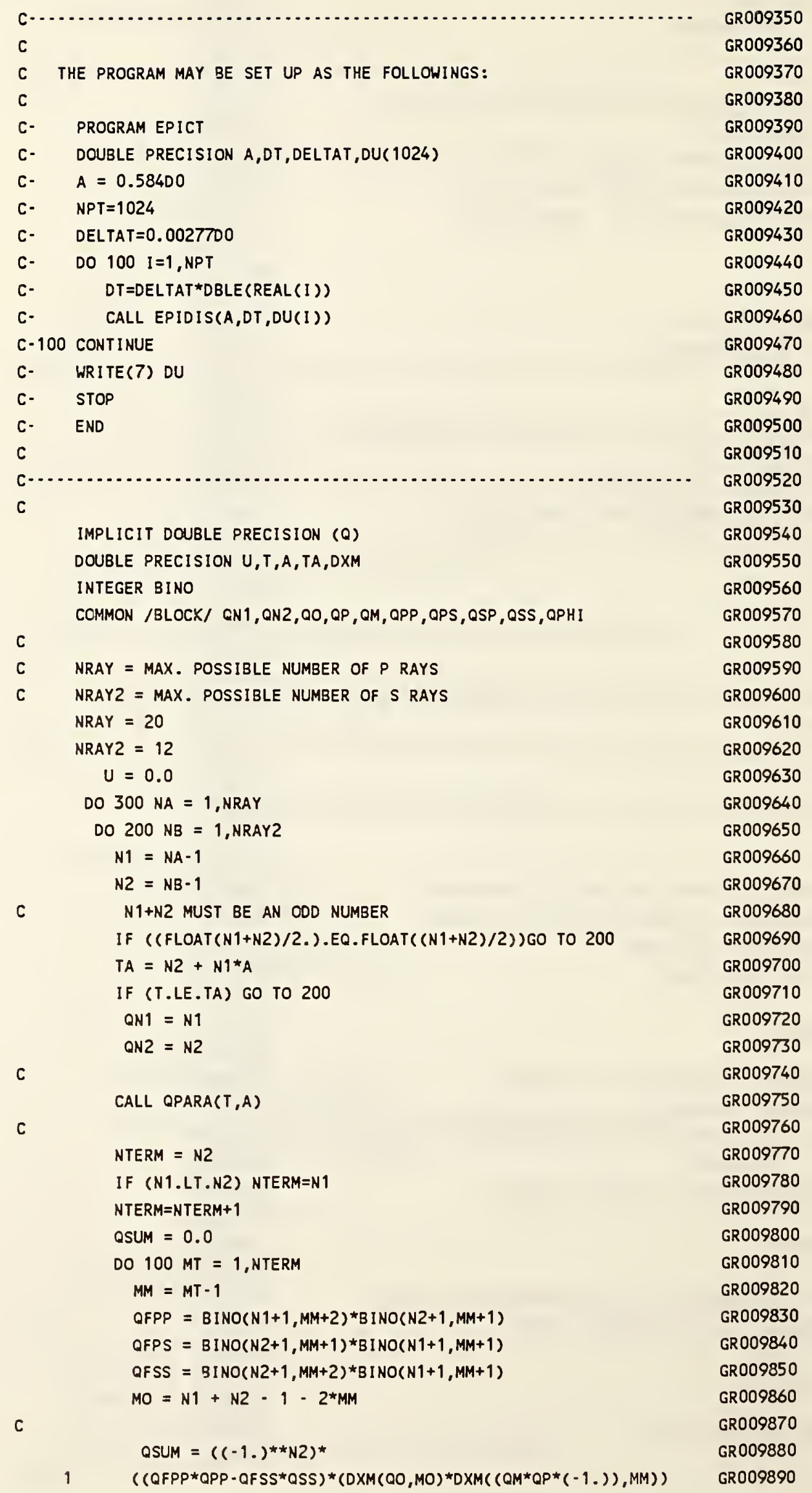




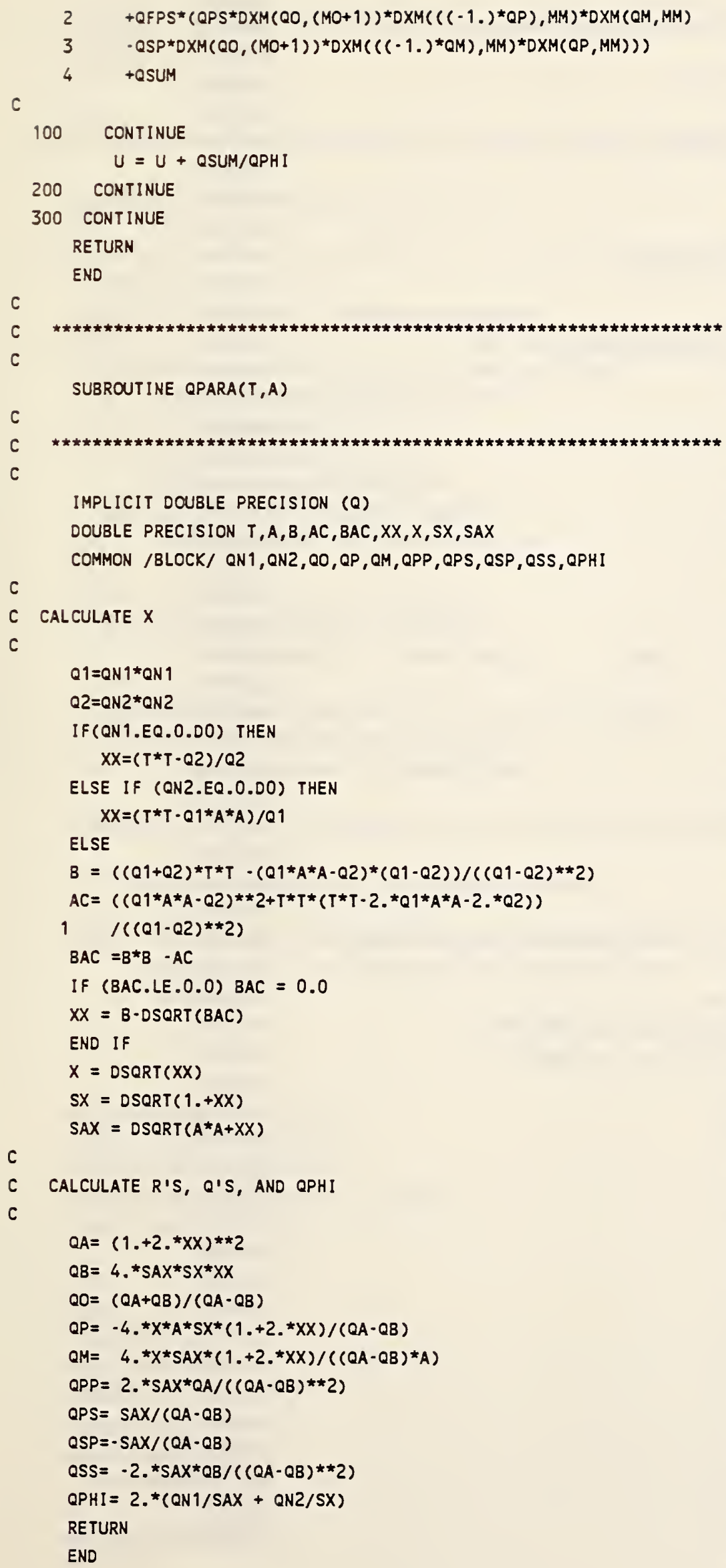




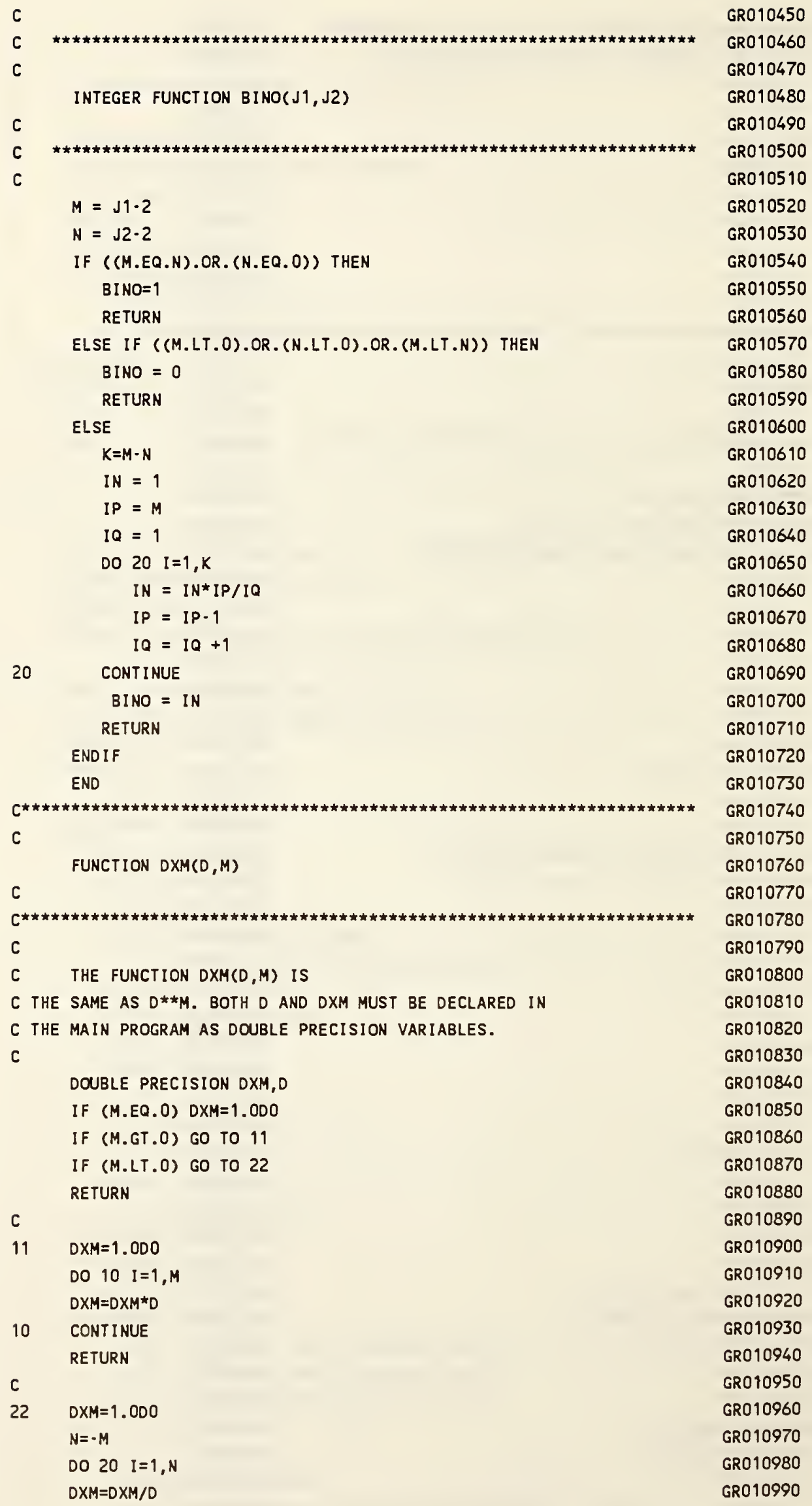


END

GR011020

GR011030

GR011040

C

SUBROUTINE SFWAVE (ALPHA, XR, TTIME, INDEX,DISPL)

GRO11050 C

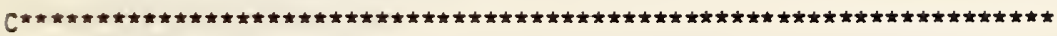

GR011060

GR011070

SFWAVE

SUBROUTINE TO COMPUTE THE SURFACE DISPLACEMENT

INPUTS:

ALPHA=RATIO OF SHEAR WAVE SPEED TO LONG. WAVE SPEED

XR =OISTANCE BETWEEN SOURCE AND DETECTOR

=ACTUAL DIST. $/ H$

TTIME = NONDIMENSIONALIZED TTIME

=ACTUAL TIME*SHEAR WAVE SPEED / $\mathrm{H}$

INDEX =SUBSCRIPT OF THE GREEN'S FUNCTION

e.g. $11,22,33,12,13$ or 131,113 , etc.

QUTPUT:

DISPL = NONDIHENSIONALIZED DISPLACEMENT

=ACTUAL DISPL. * PI * SHEAR MCOULUS * H / FORCE

NOTE:

THE PARAMETERS IN CCMMON BLKS 0,1 , \& 5 SHOULD BE ENTERED

BEFCRE THE FIRST CALL OF THIS SUBROUTINE,

1. E. CALL INIT(ALPHA, XR,ZD, INDEX) FIRST.

SUBROUITNES REGUIRED:

DSFINT, DQR, QU, COMMON, RAYRT \& INTEGRATION ROUTINES

GRO11080

GR011090

GR011100

GR011110

GRO11120

GRO11130

GR011140

GR011150

GR011160

GR011170

GR011180

GRO11190

GR011200

GR011210

GRO11220

GRO11230

GRO11240

GRO 11250

GRO11260

GR011270

GR011280

GR011290

GR011300

GR011310

REHARK: THE COMPUTATION IS DONE IN Y-PLANE, 4tN QUARD. CN A

GR011320

THREE LEGGED U-PATH TO REPLACE THE INTEGRATICN FRCY - $V$ TO V.

GR011330

GRO11340

GR011350

GRO11360

GR011370

IMPLICIT DOUBLE PRECISION (D)

GRO11380

GR011390

GR011400

GRO11410

GR011420

GR011430

GRO11440

GR011450

GR011460

GR011470

GR011480

GRO11490

GRO11500

GR011510

GRO11520

GR011530

$D P F=1.200 / D B L E(A L P H A)$

GR011540 
IF (TTIME.LE. (ALPHA*XR)) THEN

GR011550

DISPL $=0.000$

RETURN

ELSE

$D I S P L=0.00$

DISPL =DISPL+DSFING (DZERO, DMONEH, 1)

+DSFING (DV,DPF, 2)

+DSF ING (DMONEH, DZERO, 3)

GR011560

GR011570

GR011580

GR011590

GR011600

GR011610

GR011620

GR011630

GR011640

GR011650

GR011660

GR011670

DOUBLE PRECISION FUNCTION DSFING(DINIT, DEND, IPATH)

GR011680

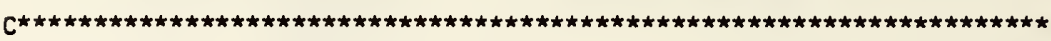

GR011690

GR011700

IMPLICIT DOUBLE PRECISION (D) GR011710

C

DOUBLE PRECISION EPS, R,E,W(50,6), FMIN, FMAX, F

GR011720

GR011730

INTEGER NINT, NMAX, KF, IFLAG

GR011740

LOGICAL RST

GR011750

C

GR011760

C

EXTERNAL DQR1, DQR2, DQR3

GR011770

C

$E P S=1.0 \cdot 8$

RST $=$.FALSE.

$\operatorname{NMAX}=50$

NINT $=1$

$H(1,1)=$ DINIT

$H(2,1)=$ DEND

GR011780

GR011790

GR011800

GR011810

GR011820

GR011830

GR011840

GR011850

GR011860

IF (IPATH.EQ.1) THEN

GR011870

CALL DGLQ1(DQR1, DINIT, DEND, EPS, R, E, NINT , RST, W, NMAX, FMIN, FMAX,

$+\quad K F$, IFLAG)

GR011880

GR011890

ELSE IF (IPATH.EQ.2)THEN

GR011900

CALL DGLQ1 (DQR2, DINIT, DEND, EPS, R, E, NINT, RST, H, NMAX, FMIN, FMAX, $+\quad$ KF, IFLAG)

GR011910

GR011920

ELSE IF (IPATH.EQ.3) THEN

GR011930

CALL DGLQ1(DQR3, DINIT, DEND, EPS, R, E, NINT, RST, H, NMAX, FMIN, FMAX, $+\quad K F$, IFLAG)

ELSE

GR011940

GR011950

GR011960

GR011970

GR011980

GR011990

GR012000

GR012010

GR012020

GR012030

GR012040

GR012050

GR012060

GR012070

IMPLICIT DOUBLE PRECISION (D)

GR012080

EXTERNAL DQR

GR012090 
DOR $1=D Q R(D L, 1)$

GR012100

RETURN

GR012110

END

GR012120

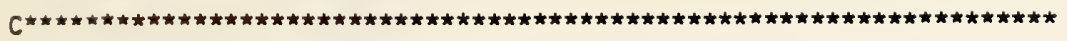

GR012130

C

DOUBLE PRECISION FUNCTION DQR2(DL)

GR012140

GR012150

$C$

GR012160

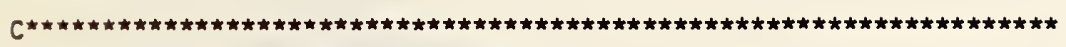

GR012170

IMPLICIT DOUBLE PRECISION (D)

EXTERNAL DQR

DQR2 $=D Q R(D L, 2)$

GR012180

GR012190

RETURN

GR012200

GR012210

END

GR012220

GR012230

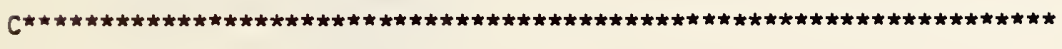

GR012240

DOUBLE PRECISION FUNCTION DQR3(DL)

GR012250

GR012260

$C$

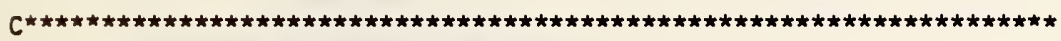

IMPLICIT DOUBLE PRECISION (D)

GR012270

GR012280

EXTERNAL DQR

DQR3=DQR (DL, 3)

GR012290

GR012300

RETURN

END

GR012310

GR012320

GR012330

GR012340

GR012350

DOUBLE PRECISION FUNCTION DOR(DL, IPATH)

GR012360

GR012370

GR012380

GR012390

IMPLICIT DOUBLE COMPLEX (Y)

GR012400

GR012410

DIMENSION YOD $(3,3), \operatorname{YUP}(3,3), \operatorname{YOK}(3), \operatorname{YFEE}(3)$

GR012420

GR012430

COMMON /BLK1/KASE, M, L, K, N1, N2,D, DTHETA

COMMON /BLK2/YD, Y, YP, YO, YRO, YRP, YRM, YDELTA, YPSOP1, YQSOP1,

GR012440

GR012450

GR012460

GR012470

GR012480

GR012490

GR012500

GR012510

GR012520

GR012530

GR012540

GR012550

GR012560

GR012570

GR012580

GR012590

GR012600

GR012610

GR012620

GR012630

GR012640 


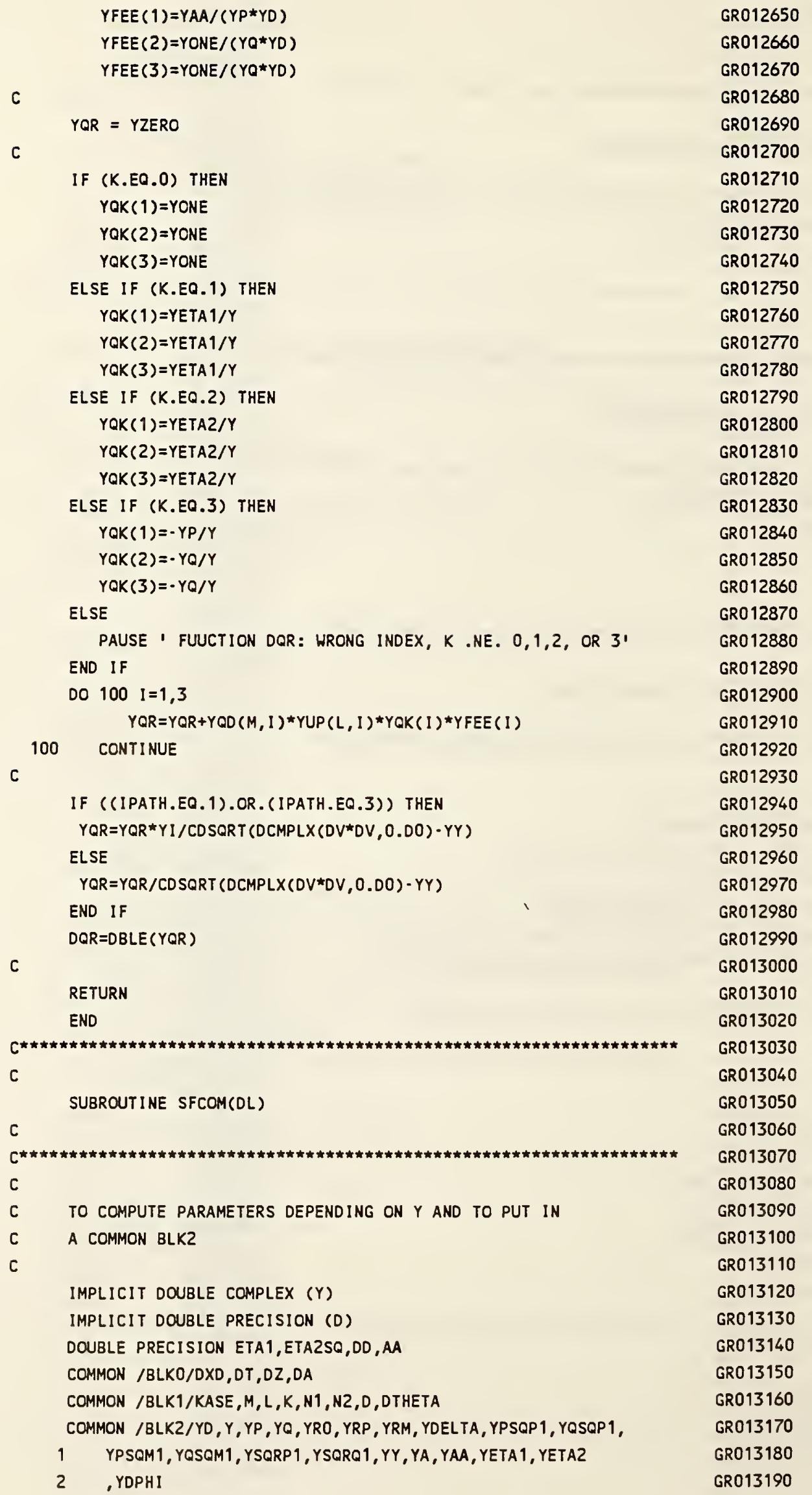


DN1=D8LE (REAL (N1))

GR013230

DN2=DBLE (REAL (N2))

GR013240

$Y Z E R O=D C M P L X(0 . D 0,0.00)$

GR013250

$Y I=D C M P L X(0.00,1.000)$

GR013260

$Y A A=D C M P L X(A A, 0 . D 0)$

GR013270

$Y A=D C M P L X(D A, 0 . D 0)$

GR013280

$Y O N E=D C M P L X(1.000,0.000)$

GR013290

YTWO=DCMPLX $(2 . D 0,0.00)$

GR013300

YFOUR=DCMPLX $(4.000,0.00)$

GR013310

GR013320

C

$Y D=-Y * D C M P L X(D T, 0 . D 0)$

GR013330

GR013340

YETA1 $=-Y / D C M P L X(D V, 0 . D O)$

GR013350

YETAZ=CDSORT (YONE - YETA1*YETA1)

GR013360

IF (DBLE(Y).LT.0.DO) YETAZ =-YETA2

GR013370

$Y Y=Y \star Y$

GRO13380

$Y P=C D S Q R T$ ( $Y A A * Y Y-Y O N E)$

GR013390

$Y Q=C D S Q R T(Y Y-Y O N E)$

GR013400

$Y P S Q P 1=Y A A * Y Y$

GR013410

YQSQP $1=Y Y$

YOSQM1 $1=Y Q \star Y Q-Y O N E$

GR013420

YPSOM1 $=Y P \star Y P-Y O N E$

GR013430

GR013440

GR013450

$Y$ SQRP $1=Y * Y A$

GR013460

$Y$ SQRQ1 $=Y$

GR013470

YDELTA $=Y Q S Q M 1 * Y Q S Q M 1+Y F O U R * Y P * Y Q$

GR013480

$Y M=(Y Q S Q M 1 * Y Q S Q M 1-Y F O U R * Y P * Y Q)$

GR013490

$Y R O=Y M / Y D E L T A$

GR013500

$Y R P=-(Y F O U R * Y P * Y O S Q M 1) /(Y A * Y D E L T A)$

GRO13510

$Y R M=(Y R O * Y R O-Y O N E) / Y R P$

GR013520

C

RETURN

GR013530

GR013540

END

GR013550

GR013560

GR013570

SUBROUTINE QU(YUP, IDUMMY)

GR013580

GR013590

GR013600

GR013610

GR013620

GR013630

GR013640

GR013650

GR013660

GR013670

GR013680

GR013690

GR013700

GR013710

GR013720

GR013730

GR013740 


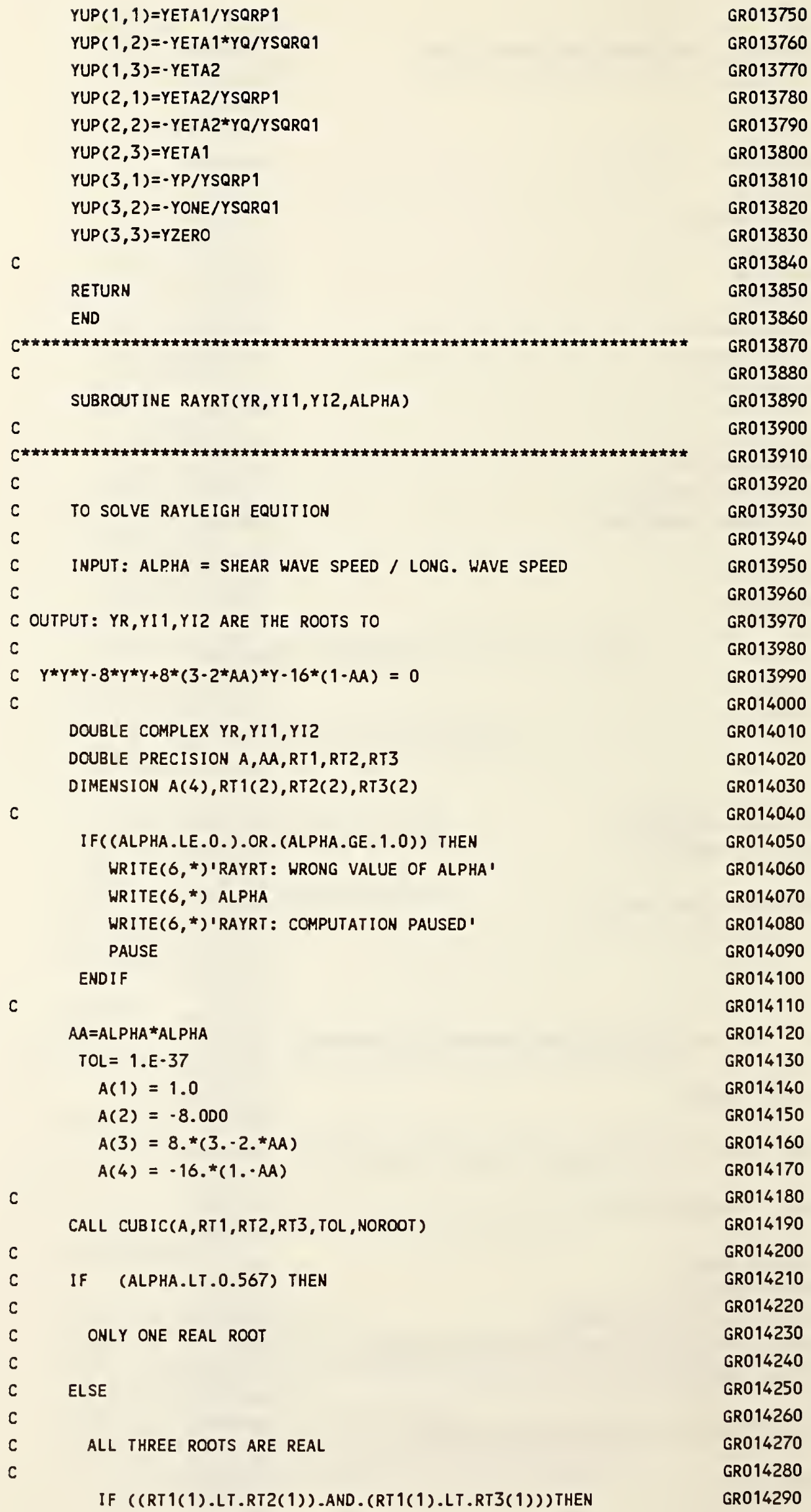


$Y R=D C M P L X(R T 1(1), R T 1(2))$

$Y I$ I $=D C M P L X(R T 2(1), R T 2(2))$

$Y I 2=D C M P L X(R T 3(1), R T 3(2))$

ELSEIF ((RT2(1).LT.RT1(1)).AND.(RT2(1).LT.RT3(1))) THEN

YI $1=D C M P L X(R T 1(1), R T 1(2))$

$Y R=D C M P L X(R T 2(1), R T 2(2))$

$Y 12=D C M P L X(R T 3(1), R T 3(2))$

ELSEIF ((RT3(1).LT.RT1(1)).AND. (RT3(1).LT.RT2(1)))THEN

$Y I 2=D C M P L X(R T 1(1), R T 1(2))$

$Y I\}=D C M P L X(R T 2(1), R T 2(2))$

$Y R=D C M P L X(R T 3(1), R T 3(2))$

ENDIF

RETURN

END$$
\text { . }
$$

C

SUBROUTINE TIMEARRI (A, R, ZD, NRAY, RCN, TA, CN)

C

C

C

C

$c$

$c$

$$
9 / 27 / 82
$$

DIMENSION $21(4), Z 2(4)$, TA(NRAY), CN(NRAY, 3), RCN(NRAY, 3)

DOUBLE COMPLEX YR, YI1, YI2

ITER $=100$

NRAY $=$ NRAY

$I=0$

$L M=1$

C $\quad z=20+0.5 ; 20$ refers to origin at the center of the plate

$z=2 D+0.5$

$21(1)=0.0$

$22(1)=2$

$21(2)=2$

$22(2)=0.0$

$21(3)=-2$

$22(3)=0.0$

$21(4)=0.0$

$z 2(4)=-2$

c

IF $(Z . E Q .1 .0) Z 2(1)=0.0$

OREVEN $=$ FLOAT $($ INT $(Z)) \star 0.5$

DO $100 \mathrm{~J}=1,21$

DO $200 \mathrm{~K}=1,12$

$J J=J \cdot 1$

$\mathrm{KK}=\mathrm{K}-1$

IF ( (K.EQ.1).AND. (J.EQ.1)) THEN IF(Z.EQ.1.0) THEN

C THREE SURFACE WAVE ARRIVALS:

$I=I+1$

$\operatorname{CN}(1,1)=-1$.

$\operatorname{CN}(1,2)=0$.

$C N(1,3)=R \star A$

$T A(I)=C N(I, 3)$

$I=I+1$
GR014300

GR014310

GR014320

GR014330

GR014340

GR014350

GR014360

GR014370

GR014380

GR014390

GR014400

GR014410

GR014420

GR014430

GR014440

GR014450

GR014460

GR014470

GR014480

GR014490

GR014500

GR014510

GR014520

GR014530

GR014540

GR014550

GR014560

GR014570

GR014580

GR014590

GR014600

GR014610

GR014620

GR014630

GR014640

GR014650

GR014660

GR014670

GR014680

GR014690

GR014700

GR014710

GR014720

GR014730

GR014740

GR014750

GR014760

GR014770

GR014780

GR014790

GR014800

GR014810

GR014820

GR014830

GR014840 


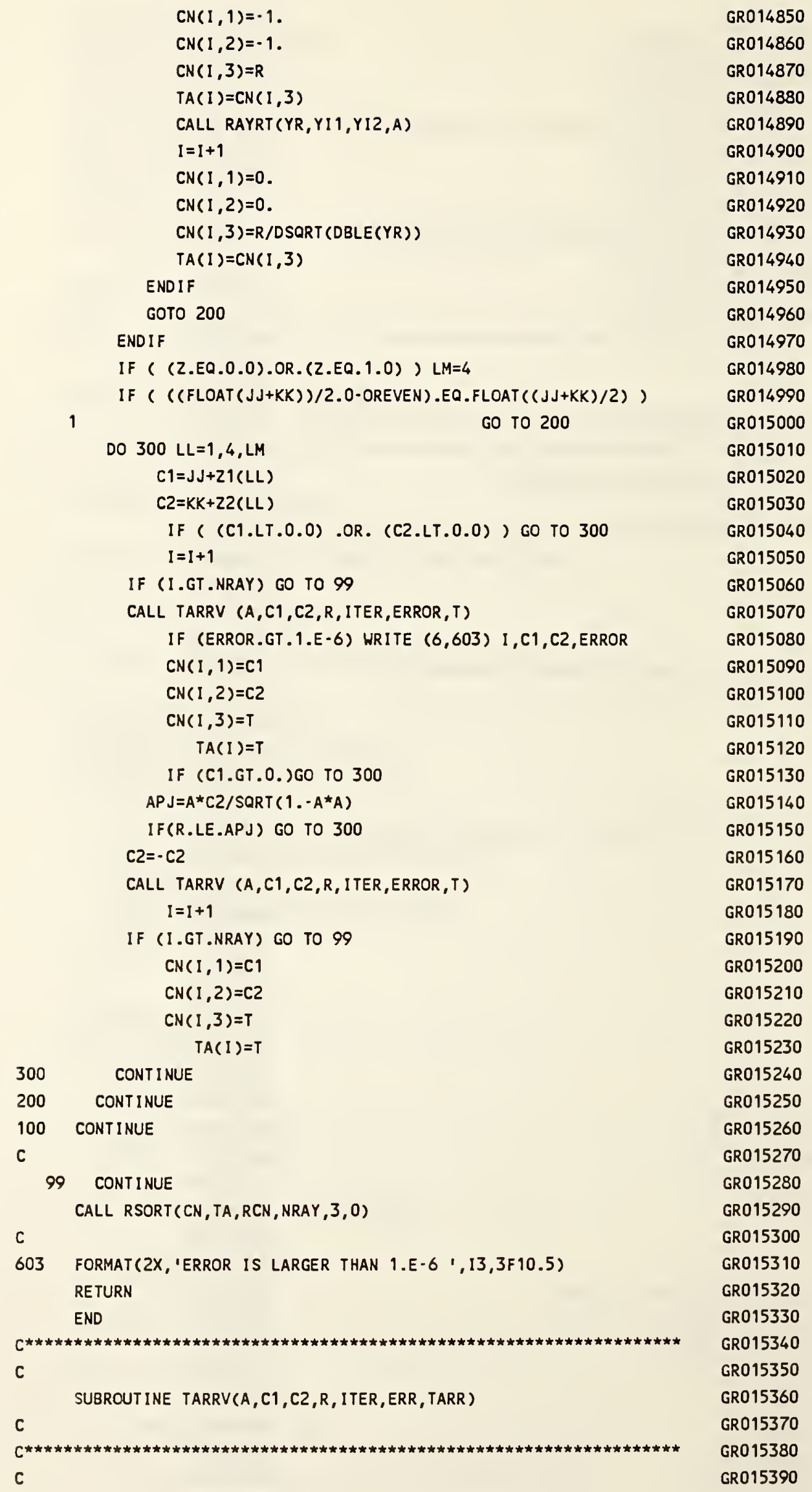


NUM $=0$

DT $1=0.1570796326794901$

GR015410

DT2 $=0.000$

GR015420

$D A=D B L E(A)$

GR015430

$D A A=D A * D A$

GR015440

$D N 1=D B L E(C 1)$

GR015450

$D N 2=D B L E(C 2)$

GR015460

GR015470

GR015480

$D R=D B L E(R)$

GR015490

IF (C1.EQ.0.0) GO TO 2

GR015500

IF (C2.EQ.0.0) GO TO 3

GRO15510

C

1 CONTINUE

GR015520

$N U M=N U M+1$

$D T=(D T 1+D T 2) / 2.000$

DAASS $=D A{ }^{*} D S I N(D T) * D S I N(D T)$

GR015530

GR015540

GR015550

GR015560

DAASS $=1.000$ - DAASS

GR015570

DCS2 =DSQR $T$ (DAASS)

GR015580

$D R T=D N 1 * D T A N(D T)+D N 2 \star D A * D S I N(D T) / D C S 2$

GR015590

C WRITE $(6,20)$ NUM,DT,DRT,C1,C2

GR015600

IF (NUM.GE.ITER) GO TO 10

GR015610

IF (DABS(DRT-DR).LE.5.D-11) GO TO 10

GR015620

IF (DRT.LE.DR) DT2=DT

GR015630

IF (DRT.GT.OR) DT $1=D T$

GR015640

GO TO 1

GR015650

c

C 20 FORMAT ('NUM= $1,13,1 \quad T=1,017.10,1 \quad R T=1,017.10,2(2 X, F 4.0)$ )

GR015660

GR015670

10 ITER $=$ NUM

$D T A R R=D A * D N 1 / D \operatorname{COS}(D T)+D N 2 / D C S 2$

GR015680

GR015690

$D E R R=D A B S(D R-D R T)$

$E R R=D E R R$

TARR = DTARR

GR015700

GR015710

GR015720

RETURN

GR015730

GR015740

GR015750

2 IF (C2.LT.0.0) GO TO 4

DTARR=DSQRT (DR*DR + DN2*DN2)

GR015760

TARR = DTARR

GR015770

$E R R=0.0$

GR015780

RETURN

GR015790

GR015800

GR015810

3 DTARR $=D S Q R T(D R * D R+D N 1 * D N 1)$ *DA

TARR = DTARR

$E R R=0.0$

GR015820

GR015830

GR015840

GR015850

GR015860

GR015870

GR015880

GR015890

GR015900

GR015910

GR015920

GR015930

C

GR015940 
SUBROUTINE CUBIC(A,RT1,RT2,RT3, TOL, NOROOT)

GR015950

C

GRO15960

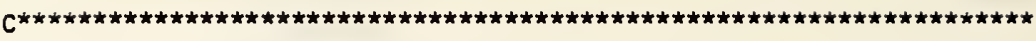

GR015970

GR015980

C THIS SUBROUTINE FINDS THE ROOTS OF A CUBIC EQUITION

GR015990

GR016000

INPUTS:

A COEFFICIENTS, A(4)

GR016010

GR016020

GR016030

OUTPUTS:

RT1,RT2,RT3 THREE ROOTS

GR016040

GR016050

RT1(1)=REAL PART OF RT1, ETC.

GR016060

GR016070

GR016080

GR016090

DOUBLE PRECISION A,B,C, RT1, RT2, RT3, ZERO, X, Y, ZZ, SOT3, RC27, ANG

GR016100

DIMENSION $A(4), R T 1(2), R T 2(2), R T 3(2), B(2), C(4)$

C

ZERO $=T O L / 10$.

SQT3=DSQRT (3.0DO)

$R C 27=1.0 / 27.0$

GR016110

GR016120

GR016130

GR016140

GR016150

GR016160

GR016170

GR016180

GR016190

GR016200

GR016210

GR016220

GR016230

GR016240

GR016250

GR016260

GR016270

GR016280

GR016290

GR016300

GR016310

GR016320

GR016330

GR016340

GR016350

GR016360

GR016370

GR016380

GR016390

GR016400

GR016410

GR016420

GR016430

GR016440

GR016450

GR016460

GR016470

GR016480

IF(RT1(1).LT.0.0) $N=1$

If (DABS(RTI(I)).LE.ZERO) GO TO 6 


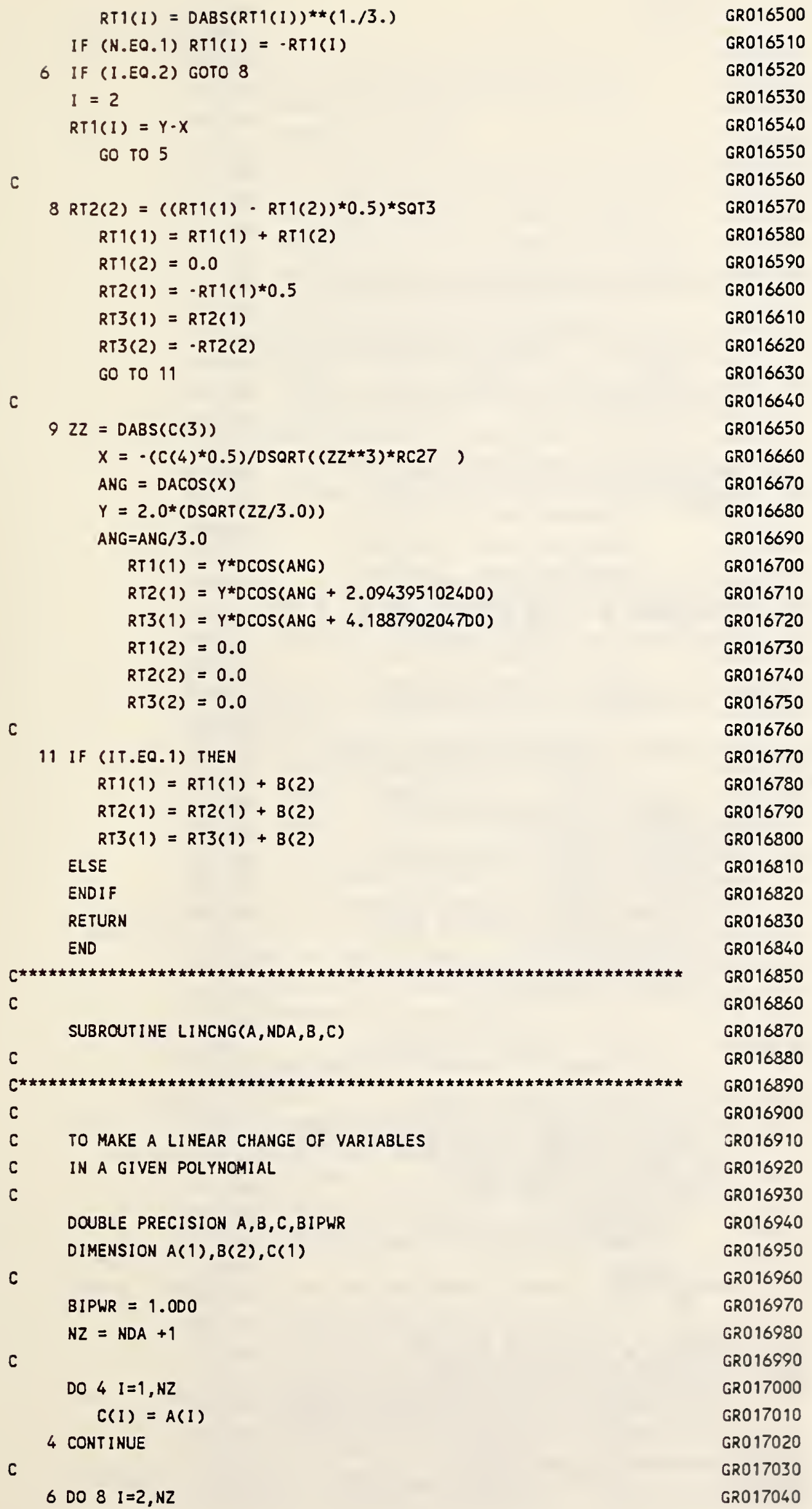


$\begin{array}{ll}C(1)=C(1)+C(1-1)^{\star} B(2) & \text { GR017050 }\end{array}$

8 CONTINUE $\quad$ GR017060

c

$C(N Z)=C(N Z) * B I P W R$

GR017070

$N Z=N Z \cdot 1$

GR017090

$B I P W R=B I P W{ }^{\star} B(1)$

GR017100

IF (NZ.GT.1) GO TO 6

GR017110

$C(1)=C(1) * B I P W R$

GR017120

RETURN

GR017130

END

GR017140$$
\text { C }
$$

C c

SUBROUT INE DGLQ1 (F , A , B , EPS, R , E, NINT , RST , W, NMAX, FMIN , FMAX, KF , I FLAG) GR017170

c

C

c

c

c

C

C

C

C

c

c

c

$\star \star \star *$

all real Variables are tYPED dOUBLE PRECISION $9 / 20 / 82 \quad N H$

GR017150

GR017160

$\star \star \star *$

$9 / 20 / 82 \quad N H$

DGLO1 IS A SUBROUTINE FOR THE AUTOMATIC EVALUATION

OF DEFINITE INTEGRALS OF A USER DEFINED FUNCTION

OF ONE VARIABLE PROVIOING FLEXIBLE USAGE.

GR017180

GR017190

GR017200

GR017210

GR017220

GR017230

GR017240

GR017250

GR017260

GR017270

GR017280

GR017290

FOR AN EASY TO USE VERSION SEE SUBROUTINE DGLQ.

GR017300

GR017310

CAPABILITIES OF DGLQ1 (IN ADDITION TO THOSE OF DGLQ)

GR017320 INCLUDE:

ABILITY TO RESTART A CALCULATION TO GREATER ACCURACY WITHOUT PENALTY...

ABILITY TO SPECIFY AN INITIAL PARTITION OF THE INTEGRATION INTERVAL...

ABILITY TO INCREASE THE WORK SPACE TO HANDLE MORE DIFFICULT PROBLEMS...

OUTPUT OF LARGEST/SMALLEST INTEGRAND VALUE FOR APPLICATIONS SUCH AS GRAPHING...

GR017330

GR017340

GR017350

GR017360

GR017370

GR017380

GR017390

GR017400

GR017410

GR017420

ARGUMENTS IN THE CALL SEQUENCE

GR017430

GR017440

F (INPUT) THE NAME OF YOUR INTEGRAND FUNCTION.

GR017450

THIS NAME MUST APPEAR IN AN EXTERNAL STATEMENT

GR017460 IN ANY PROGRAM WHICH CALLS DGLQ1.

GR017470 YOU MUST WRITE $F$ IN THE FORM

GR017480 FUNCTION $F(X)$

$F=($ EVALUATE INTEGRAND AT THE POINT $X$ )

GR017490

GR017500

GR017510

END

GR017520

GR017530

GR017540
LAGR017550

(INPUT) ENDPOINTS OF INTEGRATION INTERVAL

EPS (INPUT) ACCURACY TO WHICH THE INTEGRAL IS TO BE CALCULAGR017550
DGLQ1 WILL TRY TO ACHIEVE RELATIVE ACCURACY, GR017560

E.G. SET EPS $=.01$ FOR 2 DIGITS, .001 FOR 3, ETGR017570

(OUTPUT) THE ESTIMATE OF THE INTEGRAL

GR017580

C $E$

(OUTPUT) THE ESTIMATE OF THE ABSOLUTE ERROR IN R.

GR017590 


\begin{tabular}{|c|c|c|c|c|}
\hline C & NINT & (INPUT & & GR017600 \\
\hline$C$ & & QUTPUT) & & GR017610 \\
\hline & & & AS AN OUTPUT QUANTITY, NINT GIVES THE & GR017620 \\
\hline & & & NUMBER OF SUBINTERVALS IN THE FINAL & GR017630 \\
\hline$C$ & & & PARTITION OF $[A, B]$ & GR017640 \\
\hline$C$ & & & AS AN INPUT QUANTITY, NINT MUST BE SET TO & GR017650 \\
\hline$C$ & & & THE NUMBER OF SUBINTERVALS IN THE INITIAL & GR017660 \\
\hline & & & PARTITION OF [A,B]. FOR MOST PROBLEMS & GR017670 \\
\hline & & & THIS IS JUST 1, THE INTERVAL [A,B] ITSELF. & GR017680 \\
\hline & & & NINT IS USEFUL IF YOU WOULD LIKE TO HELP & GR017690 \\
\hline & & & DGLQ1 LOCATE A DIFFICULT SPOT ON [A,B]. & GR017700 \\
\hline & & & IN THIS REGARD NINT IS USED ALONG & GR017710 \\
\hline & & & WITH THE ARRAY W (SEE BELCW). IF YOU SET & GR017720 \\
\hline & & & NINT=1 IT IS NOT NECESSARY TO BE CONCERNED & GR017T30 \\
\hline & & & WITH $W$, EXCEPT THAT IT MUST BE DIMENSIONED... &. GR017740 \\
\hline & & & AS AN EXAMPLE OF MORE GENERAL APPLICATIONS, & GR017750 \\
\hline & & & If $[A, B]=[0,1]$ BUT THE INTEGRAND JUMPS AT 0.3 & 3GR017760 \\
\hline 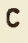 & & & IT WOULD BE WISE TO SET NINT=2 AND THEN SET & GR017770 \\
\hline 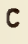 & & & (LEFT ENDPOINT) & GR017780 \\
\hline 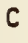 & & & (SINGULAR POINT) & GR017790 \\
\hline C & & & $H(3,1)=1.0 \quad(R I G H T$ ENDPOINT $)$ & GR017800 \\
\hline C & & & IF YOU SET NINT GREATER THAN 1, BE SURE TO & GR017810 \\
\hline C & & & CHECK THAT YOU HAVE ALSO SET & GR017820 \\
\hline & & & $W(1,1)=A \quad A N D \quad W(N I N T+1,1)=B$ & GR017830 \\
\hline$l$ & RST & (INPUT) & A LOGICAL VARIABLE (E.G. TRUE OR FALSE) & GR017840 \\
\hline C & & & SET RST=.FALSE. FOR INITIAL CALL TO DGLQ1 & GR017850 \\
\hline C & & & SET RST=.TRUE. FOR A SUBSEQUENT CALL, & GR017860 \\
\hline C & & & E.G. ONE FOR WHICH MORE ACCURACY IS & GR017870 \\
\hline & & & DESIRED (SMALLER EPS). A RESTART ONLY & GR017880 \\
\hline & & & MAKES SENSE IF THE PRECEDING CALL RETURNED & GR017890 \\
\hline & & & WITH A VALUE OF IFLAG (SEE BELOW) LESS THAN & GR017900 \\
\hline & & & ON A RESTART YOU MAY NOT CHANGE THE VALUES O & OGR017910 \\
\hline & & & ANY ARGUMENTS IN THE CALL SEQUENCE, EXCEPT E & EGR017920 \\
\hline & H(NMAX, 6 & & W IS AN ARRAY USED FOR SCRATCH STORAGE BY DGLQ1. &. GR017930 \\
\hline C & & & YOU $M U S T$ INCLUDE A DIMENSION STATEMENT IN & GR017940 \\
\hline & & & YOUR CALLING PROGRAM TO ALLOCATE THIS STORAGE. & GR017950 \\
\hline & & & THIS SHOULD BE OF THE FORM & GR017960 \\
\hline & & & DIMENSION W(NMAX,6) & GR017970 \\
\hline & & & WHERE NMAX IS AN INTEGER. AN ADEQUATE VALUE OF & GR017980 \\
\hline & & & NMAX IS 50. & GR017990 \\
\hline & NMAX & (INPUT ) & AN INTEGER EQUAL TO THE FIRST SUBSCRIPT IN THE & GR018000 \\
\hline & & & DIMENSION STATEMENT FOR THE ARRAY $W$. THIS IS & GR018010 \\
\hline & & & ALSO EQUAL TO THE MAXIMUM NUMBER OF SUBINTERVAL & LGR018020 \\
\hline & & & PERMITTED IN THE INTERNAL PARTITION OF [A,B]. & GR018030 \\
\hline 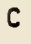 & & & A VALUE OF 50 IS AMPLE FOR MOST PROBLEMS. & GR018040 \\
\hline 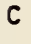 & FMIN & & & GR018050 \\
\hline 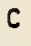 & FMAX & (OUTPUT) & THE SMALLEST AND LARGEST VALUES OF THE INTEGRAN & NGR018060 \\
\hline 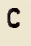 & & & WHICH OCCURRED DURING THE CALCULATION. THE & GR018070 \\
\hline 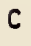 & & & ACTUAL INTEGRAND RANGE ON [A,B] MAY, OF COURS & SGR018080 \\
\hline l & & & BE GREATER BUT PROBABLY NOT BY MORE THAN $10 \%$. & GR018090 \\
\hline$l$ & KF & (OUTPUT) & THE ACTUAL NUMBER OF INTEGRAND EVALUATIONS USED & DGR018100 \\
\hline$c$ & & & BY DGLQ1 TO APPROXIMATE THIS INTEGRAL. KF & GR018110 \\
\hline$C$ & & & WILL ALHAYS BE AT LEAST 30. & GR018120 \\
\hline C & IFLAG & (OUTPUT) & TERMINATION FLAG...POSSIBLE VALUES ARE & GR018130 \\
\hline$C$ & & 0 & JORMAL COMPLETION, E SATISFIES & GR018140 \\
\hline
\end{tabular}


2 NORMAL COMPLETION, E SATISFIES GR018180 $E<E P S * D A B S(R)$

3 NORMAL COMPLETION BUT EPS WAS TOO SMALL TO GR018200 SATISFY ABSOLUTE OR RELATIVE ERROR REQUEST. GR018210

4 ABORTED CALCULATION BECAUSE OF SERIOUS ROUNDING GR018220

ERROR. PROBABLY E AND R ARE CONSISTENT. GR018230

5 ABORTED CALCULATION BECAUSE OF INSUFFICIENT STORAGR018240 $R$ AND E ARE CONSISTENT. PERHAPS INCREASING NMAGR018250

WILL PRODUCE BETTER RESULTS.

GR018260

6 ABORTED CALCULATION BECAUSE OF SERIOUS DIFFICULTIGR018270 MEETING YOUR ERROR REQUEST.

GRO 18280

7 ABORTED CALCULATION SECAUSE EITHER EPS, NINT OR NGR018290 HAS BEEN SET TO AN ILLEGAL VALUE.

GR018300

8 ABORTED CALCULATION BECAUSE YOU SET NINT>1 BUT FOGR018310 TO SET $W(1,1)=A$ AND $W(N I N T+1,1)=B \quad$ GR018320

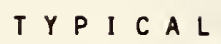


DOUBLE PRECISION A, B,E,EB, EPMACH, EPS, FMAX, FMAXL, FMAXR, FMIN, FMINL GR018700

2

DIMENSION W(NMAX,6) , FMINR, FMN, FMX, R, RAB, RABS, RAV, SIGN, T, TE

GR018710

GR018720

GR018730

EXTERNAL $F$

GR018740

LOGICAL RST, DEBUG

GR018750

DATA SIGN /-1.D0/

GR018760

GR018770

GR018780

DATA EPMACH,UFLOW/23410000000000000, $2001000000000000 /$

GR018790

GR018800

GR018810

GR 018820

EPMACH $=Y \cdot 3410000000000000$ '

GR018830

UFLOW $=Y \cdot 00100000$ '

EPMACH $=\operatorname{DBLE}\left(16^{* \star}(-13)\right)$

GR018840

UFLOW $=$ OBLE $(16 * \star(-65))$

GR018850

IF (A.EQ.B) THEN

GR018860

$R=0$.

GR018870

$E=0$.

GR018880

NINT $=0$

I FLAG $=0$

GR018890

$K F=1$

GR018900

GR018910

$F M I N=F(A)$

GR018920

FMAX $=$ FMIN

GR018930

GO TO 20

GR018940

GR018950

GR018960

IF(RST) THEN

GR018970

IF(IFLAG.LT.3) GO TO 15

GR018980

ENDIF

GO TO 20

GR018990

$\mathrm{KF}=0$

IF(EPS .LE. 0. . OR. NMAX .LE. 1 .OR. NINT .LE. O) THEN

GR019000

GR019010

IFLAG $=7$

GO TO 20

GR019020

GR019030

GR019040

GR019050

IF(NINT.EQ.1) THEN

GR019060

GR019070

$W(1,1)=A$

GR019080

$W(2,2)=B$

GR019090

$W(1,5)=A$

GR019100

$W(1,6)=B$

GR019110

$W(2,5)=A$

GR019120

$W(2,6)=B$

GR019130

$W(1,2)=A+(B-A) / 2.00$

GR019140

$W(2,1)=A+(B-A) / 2.00$

GR019150

NINT $=2$

GR019160

GR019170

IF(W(1,1).NE . A .AND. W(NINT+1,1).NE . B) THEN

GR019180

IFLAG $=8$

GR019190

GO TO 20

GR019200

ENDIF

GR019210

$W(1,5)=A$

GR019220

DO $89 \mathrm{I}=1$, NINT

GR019230

$W(1,2)=W(I+1,1)$

GR019240 


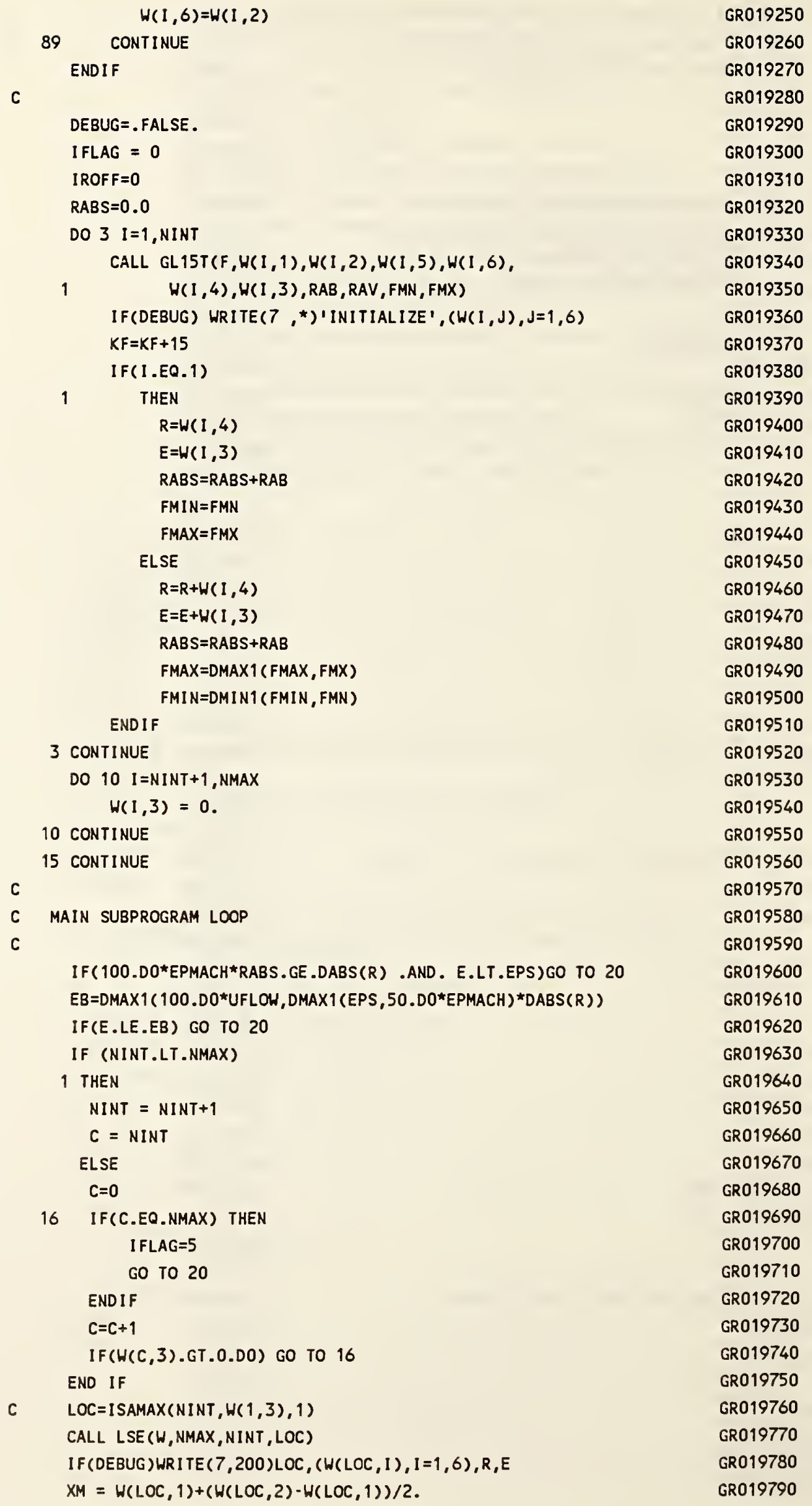


IF $((D M A X 1(D A B S(W(L O C, 1)), D A B S(W(L O C, 2)))) . G T$.

GR019800

$1((1 . D 0+100 . D 0 * E P M A C H) *(D A B S(X M)+0.10+04 * U F L O W)))$

GR019810

2 THEN

GR019820

CALL GL15T(F,W(LOC , 1), XM,W(LOC, 5), H(LOC, 6),

GR019830

TR1, TE1, RAB, RAV, FMINL, FMAXL)

GR019840

$K F=K F+15$

GKO19850

IF $\left(T E 1 . L T \cdot\left(E B^{*}(X M-W(L O C, 1)) /(B-A)\right)\right)$

GR019860

$T E I=T E I \star S I G N$

GR019870

CALL GL15T(F,XM,W(LOC, 2),W(LOC, 5),W(LOC, 6),

GR019880

GR019890

$K F=K F+15$

TR2, TE2, RAB, RAV, FMINR, FMAXR)

GR019900

FMIN $=D M I N 1$ ( FMIN , FMINL, FMINR)

GR019910

FMAX =DMAX1 ( $F M A X, F M A X L$, FMAXR)

GR019920

IF $\left(T E 2 . L T \cdot\left(E B^{*}(H(L O C, 2)-X M) /(B-A)\right)\right)$ TEZ=TE2*SIGN

GR019930

$T E=D A B S(W(L O C, 3))$

$T R=H(L O C, 4)$

GR019940

$H(C, 3)=T E 2$

GR019950

GR019960

$W(C, 4)=T R 2$

GR019970

$W(C, 1)=X M$

GR019980

$W(C, 2)=W(L O C, 2)$

GR019990

$W(C, 5)=H(L O C, 5)$

GR020000

$W(C, 6)=W(L O C, 6)$

GR020010

$H(L O C, 3)=$ TE 1

GR020020

$W(\operatorname{LOC}, 4)=T R 1$

GR020030

$W(L O C, 2)=X M$

GR020040

IF(DEBUG)WRITE $(7,200) C,(H(C, K), K=1,6)$

GR020050

IF (DEBUG)WRITE $(7,200)$ LOC, $(W(L O C, K), K=1,6)$

GR020060

$E=E-T E+(D A B S(T E 1)+D A B S(T E 2))$

GR020070

$R=R-T R+(T R 1+T R 2)$

IF (DEBUG)WRITE ( 7 , *)NINT, R, E

IF (DABS (DABS (TE1)+DABS (TE2) - TE). LT..00100ะTE) THEN

GR020080

GR020090

GR020100

IROF $F=I R O F F+1$

IF(IROFF.GE.10) THEN

GR020110

GR020120

I FLAG $=4$

GO TO 20

GR020130

GR020140

GR020150

ENDIF

GR020160

END I $F$

ELSE

IF (EB.GT.H(LOC, 3))

GR020170

GRO20180

GR020190

GR020200

GR020210

GRO20220

GR020230

GR020240

END IF

GO TO 20

GR020250

GR020260

GR020270

GR020280

GR020290

GR020300

GR020310

GR020320

GR020330

GR020340 
I $F L A G=2$

GR020350

IF(E.GT.EPS .AND. E.LT.T)RETURN GR020360

I FLAG $=1$

IF(E.LT.EPS .AND. E.GT.T)RETURN

GR020370

GR020380

IFLAG $=0$

GR020390

RETURN

200 FORMAT (I4,8(E11.3))

GR020400

GR020410

END

GR020420

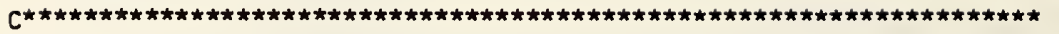

GR020430

$c$

SUBROUTINE LSE (WORK, NMAX, NI, LOC)

GR020440

GR020450

C

C

GR020460

GR020470

GR020480

THIS SUBPROGRAM FINDS THE CELL IN THE WORK AREA

GR020490

GR020500

GR020510

GR020520

GR020530

DOUBLE PRECISION ERROR, WORK

GR020540

DIMENSION WORK (NMAX, 6)

GR020550

GR020560

GR020570

GR020580

GR020590

GR020600

GR020610

GR020620

GR020630

GR020640

GR020650

GR020660

GR020670

END IF

20 CONTINUE

GR020680

GR020690

GR020700

GR020710

GR020720

GR020730

GR020740

GR020750

GR020760

GR020770

GR020780

GR020790

GR020800

GR020810

GR020820

GR020830

GR020840

GR020850

GR020860

GR020870

PARAMETERS

GR020880

ON ENTRY

GR020890 
GR021000

GR021010

GR021020

GR021030

GR021040

GR021050

GR021060

GR021070

GR021080

GR021090

GR021100

GR021110

GR021120

GRO21130

GRO21140

GRO21150

GR021160

GR021170

GR021180

GR021190

GR021200

GR021210

GR021220

GRO21230

GRO21240

GR021250

GR021260

GRO21270

GR021280

GRO21290

GR021310

GRO21320

GR021330

GR021340

GR021350

GR021360

GR021370

GR021380

GR021390

GR021600 
* WG, WGK, XGK

GR021450

DOUBLE PRECISION XL, XR, CENTR, ABSC, U, FMAX, FMIN, PHI, PHIP GR021460

INTEGER $J$, JTW, JTWMI GR021470

c

DIMENSION FV1(7), FV2(7), WG(4),WGK(8), XGK(8) GR021490

THE ABSCISSAE AND WEIGHTS ARE GIVEN FOR THE INTERVAL $(-1,1)$ BECAUSE OF SYMMETRY ONLY THE POSITIVE ABSCISSAE AND THEIR CORRESPONDING WEIGHTS ARE GIVEN.

XGK - ABSCISSAE OF THE 15-POINT KRONROD RULE XGK(2), XGK(4), ... ABSCISSAE OF THE 7-POINT GAUSS RULE XGK(1), XGK(3), . ABSCISSAE WHICH ARE OPTIMALLY GRO21580 ADDED TO THE 7-POINT GAUSS RULE

WGK - WEIGHTS OF THE 15-POINT KRONROD RULE

WG - WEIGHTS OF THE 7-POINT GAUSS RULE

DATA XGK(1), XGK(2), XGK(3), XGK(4),XGK(5), XGK(6), XGK(7), XGK(8)/

* $0.99145537112081260+00,0.94910791234275850+00$,

* $0.86486442335976910+00,0.7415311855993944 D+00$,

* $\quad 0.58608723546769110+00,0.4058451513773972 D+00$,

* $\quad 0.20778495500789850+00,0.00+00$

DATA $\operatorname{WGK}(1), \operatorname{WGK}(2), \operatorname{WGK}(3), \operatorname{WGK}(4), \operatorname{WGK}(5), \operatorname{WGK}(6), \operatorname{WGK}(7), \operatorname{WGK}(8)$ /

* $\quad 0.22935322010529220-01,0.63092092629978550-01$,

* $\quad 0.1047900103222502 D+00,0.14065325971552590+00$,

* $\quad 0.16900472663926790+00,0.19035057806478540+00$,

* $\quad 0.20443294007529890+00,0.20948214108472780+00$ /

DATA WG(1),WG(2), WG(3), WG(4)/

* $\quad 0.12948496616886970+00,0.27970539148927670+00$,

* $\quad 0.38183005050511890+00,0.41795918367346940+00 /$

DATA EPMACH,UFLOW/Z3410000000000000,20010000000000000/

PHI (U) $=X R \cdot(X R \cdot X L) * U \star U *(2 . D O * U+3 . D 0)$

$P H I P(U)=-6 . D O * U *(U+1 . D 0)$

LIST OF MAJOR VARIABLES

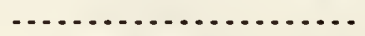

CENTR - MID POINT OF THE INTERVAL

HLGTH - HALF-LENGTH OF THE INTERVAL

ABSC - ABSCISSA

FVAL* - FUNCTION VALUE

RESG - R OF THE 7-POINT GAUSS FORMULA

RESK - $R$ OF THE 15-POINT KRONROD FORMULA

RESKH - APPROXIMATION TO THE MEAN VALUE OF F OVER $(A, B)$, I.E. TO $I /(B-A)$

MACHINE DEPENDENT CONSTANTS

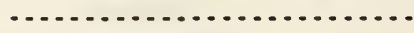

EPMACH IS THE LARGEST RELATIVE SPACING.

UFLOW IS THE SMALLEST POSITIVE MAGNITUDE.
GR021500

GR021510

GR021520

GR021530

GR021540

GR021550

GR021560

GR021570

GR021590

GR021600

GR021610

GR021620

GR021630

GR021640

GR021650

GR021660

GR021670

GR021680

GR021690

GR021700

GR021710

GR021720

GR021730

GR021740

GR021750

GR021760

GR021770

GR021780

GR021790

GR021800

GR021810

GR021820

GR021830

GR021840

GR021850

GR021860

GR021870

GR021880

GR021890

GR021900

GR021910

GR021920

GR021930

GR021940

GR021950

GR021960

GR021970

GR021980

GR021990 
GR022060

GR022070

GR022080

GR022090

GR022100

GR022110

GR022120

GR022130

GR022140

GR022150

GR022160

GR022170

GR022180

GR022190

GR022200

GR022210

GR022220

GR022230

GR022240

GR022250

GR022260

GR022270

GR022280

GR022290

GR022300

GR022310

GR022320

GR022330

GR022340

GR022350

GR022360

GR022370

GR022380

GR022390

GR022400

GR022410

GR022420

GR022430

GR022440

GR022450

GR022460

GR022470

GR022480

GR022490

GR022500

GR022510

GR022520

GR022530

GR022540 
RESK $=$ RESK+WGK $(J T W M 1) \star F S U M$

GR022550

$R A=R A+W G K(J T W M 1) *(D A B S(F V A L 1)+D A B S(F V A L 2)) \quad$ GR022560

15 CONTINUE

RESKH $=$ RESK*0.50+00

GR022570

GR022580

RASC $=$ WGK $(8) * D A B S(F C \cdot R E S K H)$

GR022590

DO $20 \mathrm{~J}=1,7$

RASC $=\operatorname{RASC}+W G K(J) *(D A B S(F V 1(J) \cdot \operatorname{RESKH})+D A B S(F V 2(J)-\operatorname{RESKH}))$

GR022600

GR022610

20 CONTINUE

$R=$ RESK*HLGTH

GR022620

$R A=R A{ }^{\star} D H L G T H$

GR022630

RASC = RASC*DHLGTH

$A E=D A B S((R E S K-R E S G) * H L G T H)$

I F (RASC. NE . 0.0D+00.AND.AE.NE. 0.0D+00)

GR022640

GR022650

GR022660

GR022670

* $\quad A E=$ RASC*DMIN1 $(0.10+01$,

* $(0.2 D+03 * A E / R A S C) \star * 1.5 D+00)$

IF (RA.GT.UFLOW/(0.5D+02*EPMACH $))$ AE = DMAX1

* ((EPMACH*0.50+02)*RA,AE)

RETURN

END

GR022680

GR022690

GR022700

GR022710

GR022720

GR022730

GR022740

GR022750

GR022760

SUBROUTINE QUADRT (A, ROOT1, ROOT2, TOL, NOROOT)

GR022770

GR022780

GR022790

GR022300

GR022810

GR022820

GR022830

GR022840

DOUBLE PRECISION A, ROOT $1, R O O T 2, X, Y, Z, W, Z E R O$

GR022850

GR022860

GR022870

GR022880

GR022890

GR022900

GR022910

GR022920

GR022930

GR022940

GR022950

GR022960

GR022970

GR022980

GR022990

GR023000

GR023010

GR023020

GR023030

GR023040

GR023050

GR023060

GR023070

GR023080

GR023090 


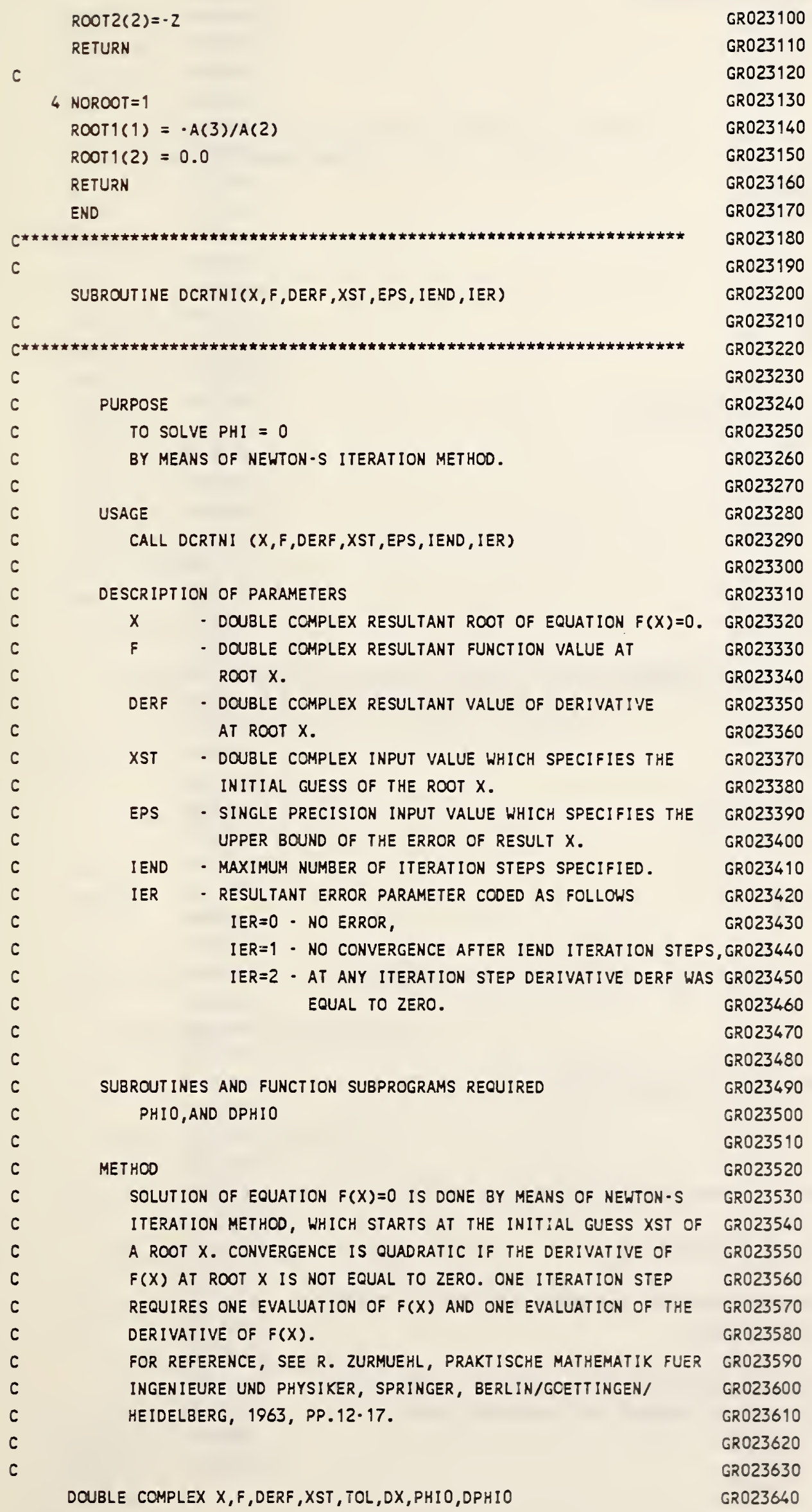


DCUBLE PRECISION A,DTOL,DTOLF

GR023650

EXTERNAL PHIO,DPHIO

GR023660

C PREPARE ITERATION

GR023670

IER $=0$

GR023680

$X=X S T$

GR023690

TOL $=X$

GR023700

$F=P H I O(T O L)$

GR023710

DERF $=$ DPHIO(TOL)

GR023720

DTOLF $=100 . D D^{\star E P S}$

GR023730

C

GR023740

c

C START ITERATION LOOP

GR023750

DO $6 \mathrm{I}=1$, IEND

GR023760

IF $(\operatorname{CDABS}(F)) 1,7,1$

GR023770

GR023780

C

C EQUATION IS NOT SATISFIED BY $X$

GR023790

1 IF (CDABS (DERF )) 2,8,2

GR023800

C

C ITERATION IS POSSIBLE

GR023810

GR023820

GR023830

$2 D X=F / D E R F$

GR023840

$X=X-D X$

GR023850

$\mathrm{TOL}=\mathrm{X}$

GR023860

$F=P H I O(T O L)$

GR023870

DERF $=$ DPHIO(TOL)

GR023880

GR023890

GR023900

GR023910

GR023920

GR023930

GR023940

GR023950

GR023960

GR023970

GR023980

GR023990

GR024000

GR024010

GR024020

GR024030

GR024040

GR024050

GR024060

GR024070

GR024080

GR024090

GR024100

GR024110

GR024120

GR024130

GR024140

GR024150

GR024160

GR024170

GR024180

GR024190 


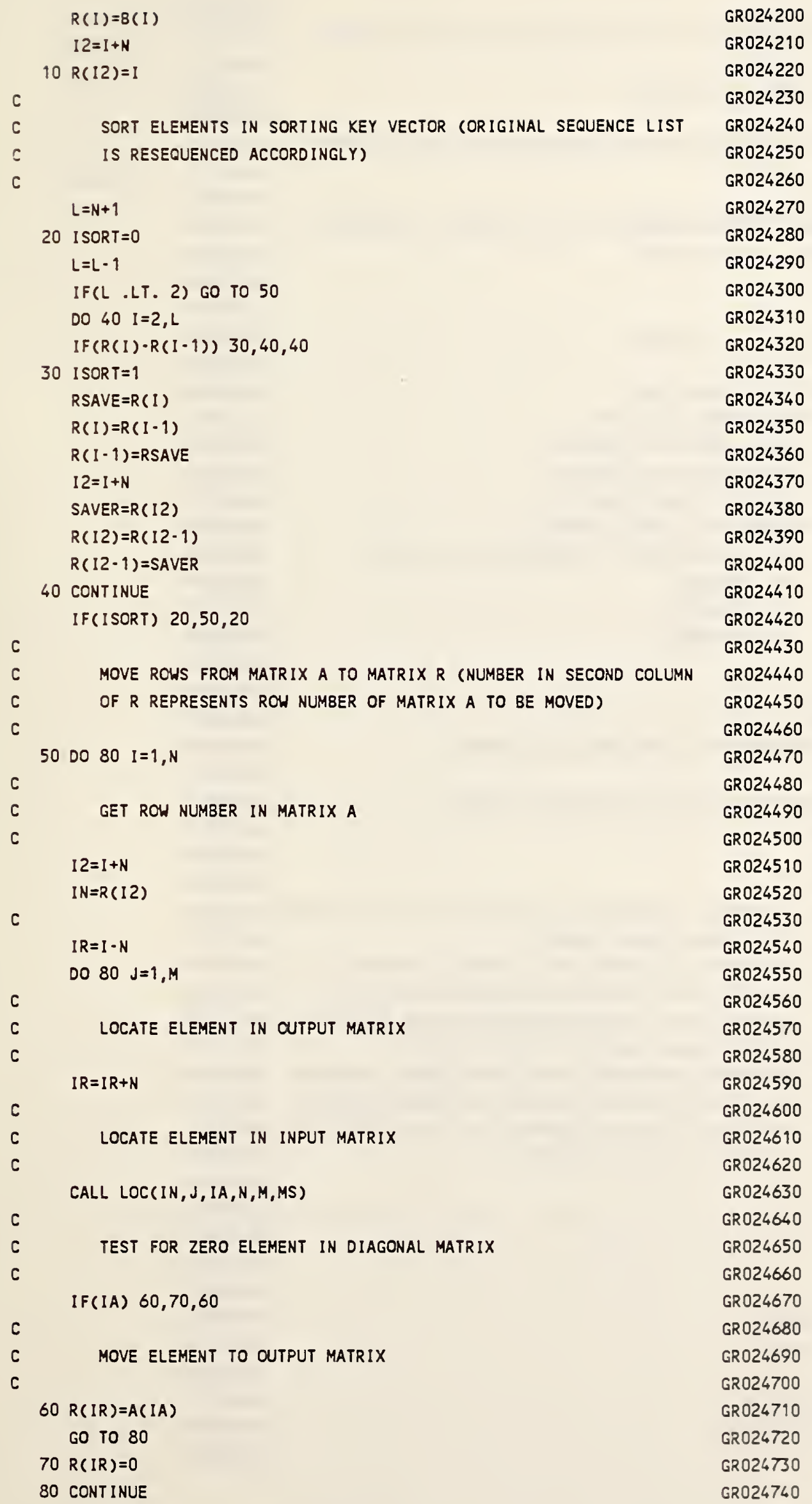


C

C

C

C

c

C

C

C

I $X=I$

$J X=J$

I $F(M S-1) \quad 10,20,30$

$10 I R X=N^{*}(J X-1)+I X$ GO TO 36

20 IF $(I X-J X) 22,24,24$

$22 I R X=I X+(J X * J X-J X) / 2$ GO TO 36
GR024800

GR024810

GR024820

GR024830

GR024840

GR024850

GR024860

GR024870

GR024880

GR024890

GR024900

GR024910

GR024920

GR024930

GR024940

GR024950

GR024960

GR024970

GR024980

GR024990

GR025000

GR025010

GR025020

GR025030

GR025040

GR025050

GR025060

GR025070

GR025080

MS $=0$ SUBSCRIPT IS COMPUTED FOR A MATRIX WITH N*M ELEMENTS GR025090 IN STORAGE (GENERAL MATRIX)

GR025 100

MS $=1$ SUBSCRIPT IS COMPUTED FOR A MATRIX WITH $N^{*}(N+1) / 2$ IN GR025110 STORAGE (UPPER TRIANGLE OF SYMMETRIC MATRIX). IF GR025120 ELEMENT IS IN LOWER TRIANGULAR PORTION, SUBSCRIPT IS GR025130 CORRESPONDING ELEMENT IN UPPER TRIANGLE. GR025140

MS $=2$ SUBSCRIPT IS COMPUTED FOR A MATRIX WITH N ELEMENTS GR025150 IN STORAGE (DIAGONAL ELEMENTS OF DIAGONAL MATRIX). GR025160 IF ELEMENT IS NOT ON DIAGONAL (AND THEREFORE NOT IN GR025170 STORAGE), IR IS SET TO ZERO. GR025180 GR025190 GR025200 GR025210 GR025220 GR025230 GR025240 GR025250 GR025260 GR025270 GR025280 GR025290 
$24 I R X=J X+(I X * I X-I X) / 2$

GR025300 GO TO 36

GR025310

30 I $R X=0$

GR025320

I $f(I X-J X) \quad 36,32,36$

GR025330

32 I $R X=I X$

GR025340

$36 I R=I R X$

GR025350

RETURN

GR025360

END

GR025370 

NBS-114A (AEV. $2.8 C$ )

U.S. OEPT. OF COMM.

BIBLIOGRAPHIC DATA

SHEET (see instructions)

1. PUBLICATION OR REPORT NO.

NBSIR/85-3234

2. Performing Organ. Report Nof 3. Publication Date

NOVENBER 1985

4. TITLE AND SUBTITLE

Dynamic Green's Functions of An Infinite Plate - A Computer Program

5. AUTHOR(S)

Nelson N. Hsu

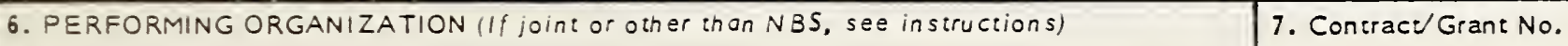

NATIONAL BUREAU OF STANDARDS

DEPARTMENT OF COMMERCE

Tontracd Grant No.

WASHINGTON, D.C. 20234

9. SPONSORING ORGANIZATION NAME AND COMPLETE ADDRESS (Street, City, State, ZIP)

8. Type of Report \& Period Covered

10. SUPPLEMENTARY NOTES

Document describes a computer program; SF-185, FIPS Software Summary, is attached.

11. ABSTRACT (A 200-word or less factual summary of most significant information. If document includes a significan: bibliography or literature survey, mention it here)

This report is a FORTRAN program to compute the Green's functions of an infinite plate. The Green's function, $G_{i j}(\underline{\xi}, \underline{x}, t)$, is defined as the ith component of the displacement at $x$ due to a point force of step-function time dependency acting at $\underline{\xi}$ in the jth direction initiated at $t=0$. The Green's function is the fundamental solution of the transient elastic wave propagation problen. In general, the displacement field $\underline{u}(\underline{\xi}, \underline{x}, t)$ at $\underline{x}$ due to a point force of arbitrary time dependence acting at $\underline{\xi}$ can $\bar{b} e$ computed $\bar{b} y$ a convolution integration; i.e.,

$$
u_{i}(\underline{\xi}, \underline{x}, t)=\int_{0}^{\infty} G_{i j}(\underline{\xi}, \underline{x}, t) f_{j}(\tau-t) d \tau .
$$

Here, $G_{i j}$ is the time derivative of $G_{i j}$ and $f_{j}(t)$ is the point force component of arbitrary time dependence acting in the jth direction (summation over

repeated indices is used). Displacement due to point dipoles or couple forces can be represented by the spatial derivatives of $G_{j j}$. Displacement produced by a dynamic force distributed over a finite area can lalso be computed by numerical integration using the Green's function as the kernel of the integral over the finite area.

12. KEY WORDS (Six to twelve entries; alphabetical order; copitalize only proper names; and separate key words by semicolons, Acoustic emission; dynamic displacement due to point impact; Green's function; ray theory; transient wave propagation; waveform analysis

13. AVAILABILITY

$\bar{X}$ Unlimited

For Official Distribution. Do Not Release to NTIS

Order From Suderintendent of Documents, U.S. Government Printing Office, Washington. D.C. 20402.

14. NO. OF

PRINTED PAGES

66

15. Peice

[X Order From National Technical Information Service (NTIS), Springrield, VA. 22161 


